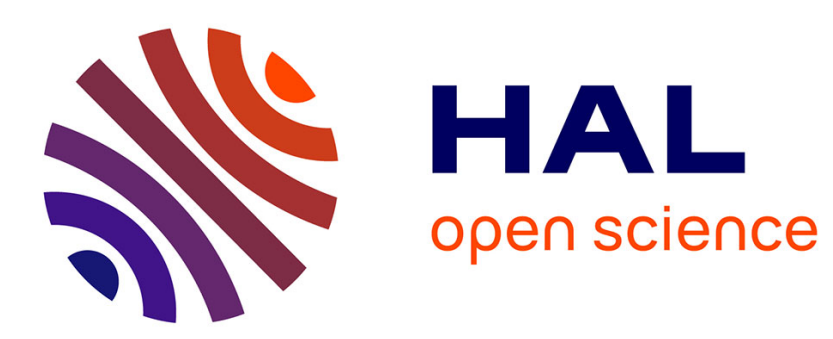

\title{
Semidefinite programming for optimizing convex bodies under width constraints
}

Terence Bayen, Didier Henrion

\section{To cite this version:}

Terence Bayen, Didier Henrion. Semidefinite programming for optimizing convex bodies under width constraints. Optimization Methods and Software, 2012, 27 (6), pp.1073-1099. 10.1080/10556788.2010.547580 . hal-00495031

\section{HAL Id: hal-00495031 \\ https://hal.science/hal-00495031}

Submitted on 24 Jun 2010

HAL is a multi-disciplinary open access archive for the deposit and dissemination of scientific research documents, whether they are published or not. The documents may come from teaching and research institutions in France or abroad, or from public or private research centers.
L'archive ouverte pluridisciplinaire HAL, est destinée au dépôt et à la diffusion de documents scientifiques de niveau recherche, publiés ou non, émanant des établissements d'enseignement et de recherche français ou étrangers, des laboratoires publics ou privés. 


\title{
Semidefinite programming for optimizing convex bodies under width constraints
}

\author{
Térence Bayen $^{1}$, Didier Henrion ${ }^{2,3}$
}

June 25, 2010

\begin{abstract}
We consider the problem of minimizing a functional (like the area, perimeter, surface) within the class of convex bodies whose support functions are trigonometric polynomials. The convexity constraint is transformed via the Fejér-Riesz theorem on positive trigonometric polynomials into a semidefinite programming problem. Several problems such as the minimization of the area in the class of constant width planar bodies, rotors and space bodies of revolution are revisited. The approach seems promising to investigate more difficult optimization problems in the class of three-dimensional convex bodies.
\end{abstract}

Keywords: convexity, optimization, semi-definite programming, support function.

AMS Subject Classification: 49M27,90C22,52C05

\section{Introduction}

Many optimization problems involve convexity constraints. Such constraints naturally appear in the framework of geometric optimization problems, where the underlying admissible class is the set of convex bodies in Euclidean space. This paper aims to tackle the issue of a certain class of planar geometric optimization problems via semidefinite programming.

A convex body can be parametrized by its support function defined by homogeneity on the unit hypersphere. The convexity constraint amounts to the non-negativity of the principal radii of curvature of the boundary of the body (if they are defined). For the planar case, the convexity constraint is equivalent to the non-negativity of the radius of curvature of the boundary of the body, easily expressed via the support function. A reformulation of the convexity constraint allows the use of the calculus of variation and optimal control theory to treat

\footnotetext{
${ }^{1}$ Institut de Mathématiques et de Modélisation de Montpellier, Université Montpellier 2, Case Courrier 051, Place Eugène Bataillon, 34095 Montpellier Cedex, France. terence. bayen@math.univ-montp2.fr

${ }^{2}$ CNRS; LAAS; 7 avenue du colonel Roche, F-31077 Toulouse, France; Université de Toulouse; UPS, INSA, INP, ISAE; LAAS; F-31077 Toulouse, France. henrion@laas.fr

${ }^{3}$ Faculty of Electrical Engineering, Czech Technical University in Prague, Technická 4, CZ-16626 Prague, Czech Republic. henrion@fel.cvut.cz
} 
several optimization problems for two- and three-dimensional bodies, such as the minimization of the area within the class of constant width bodies and rotors, see [3, 4, 5, 6, 10].

The approach which is chosen in this paper significantly differs from the previous ones. We consider planar convex bodies which are parametrized by their support function modeled by a truncated Fourier series. The convexity constraint becomes a trigonometric positivity constraint, and it turns out that it can be formulated as a linear decision problem in the convex cone of positive semidefinite matrices, with decision variables the Fourier coefficients of the support function.

Several optimization problems involving convex bodies can be formulated via this method. For example, the problem of minimizing the area within the class of constant width bodies and rotors [1] can be gathered into this form. The area is a concave quadratic functional with respect to Fourier coefficients, and the problem of minimizing the area under additional width constrains amounts to solving a nonconvex semidefinite programming problem (concave quadratic objective function, convex semidefinite constraints), for which we can use a public-domain Matlab implementation of a penalty augmented Lagrangian method. In this paper, we mainly deal with convex bodies satisfying width constraints, and the functional to be minimized is quadratic concave (area) or linear (perimeter) with respect to the decision variables.

The paper is organized as follows. In section 2, well-known results on the parametrization of a planar convex body by its support function are recalled. Then, the reformulation of the convexity constraint into a semidefinite programming (SDP) problem is presented. In the third section, we solve numerically (using YALMIP and PENBMI, see [15]) several optimization problems:

- minimization of the area within the planar constant width bodies (section 3.1),

- minimization of the area within the class of rotors (section 3.2),

- minimization of the area within three dimensional constant width bodies of revolution (section 3.3),

- minimization of the area and the perimeter within the class of convex bodies with given minimal and maximal width (section 3.4).

The numerical experiments allow to retrieve known results with closed-form solutions and to validate the method (problems of sections 3.1, 3.2 and 3.3). In section 3.4, the method is used to derive new results and to find numerical candidates for optimality (extrema of the perimeter or area) in the class of planar convex bodies for which the minimal width is 1 and the maximal width is 2 . The last section 1 is devoted to the proof of the convergence of the method (for problem of sections 3.1,3.2) when the number of Fourier coefficients of the truncated Fourier series goes to infinity. More precisely, we prove that the value of the functional evaluated for a minimizer of the finite-dimensional problem converges to the value of the functional for the global optimization problem (Theorem 4.1). 


\section{General results}

\subsection{Parametrization of a convex body by its support function}

In this section we recall classical results on the parametrization of a convex set by its support function. Let $K$ be a body, that is a non-empty compact set of $\mathbb{R}^{n}$, and let $K$ be convex. The support function $\sigma$ of $K$ is the map defined by

$$
u \in \mathbb{R}^{n} \backslash\{0\} \mapsto \sigma(u)=\max _{x \in K} x \cdot u
$$

where the dot denotes the scalar product. The support function can be equivalently defined on the unit hypersphere $\mathbb{S}^{n-1}$ by homogeneity. It is standard that $\sigma$ is of class $C^{1}$ if and only if $K$ is strictly convex, see [20]. In this paper we consider convex bodies of $\mathbb{R}^{2}$, that is $n=2$. The parametrized support function $p$ of $K$ is then defined by

$$
\theta \in \mathbb{R} \mapsto p(\theta)=\sigma(\cos \theta, \sin \theta) .
$$

By extension, function $p$ will be called support function of $K$. If $K$ is strictly convex, its boundary $\partial K$ can be described by

$$
\left\{\begin{array}{l}
x(\theta)=p(\theta) \cos \theta-p^{\prime}(\theta) \sin \theta \\
y(\theta)=p(\theta) \sin \theta+p^{\prime}(\theta) \cos \theta
\end{array}\right.
$$

where the prime denotes differentiation. If $p$ is of class $C^{1,1}$ (that is $p^{\prime}$ is Lipschitz), $p^{\prime \prime}$ exists almost everywhere (a.e.) by Rademacher's theorem. The quantity $\rho=p+p^{\prime \prime}$ is the radius of curvature of the boundary of $K$ and we have

$$
\rho=p+p^{\prime \prime} \geq 0
$$

by convexity of $K$. Notice that $p$ and $\rho$ are related by

$$
p(\theta)=\int_{0}^{\theta} \rho(t) \sin (\theta-t) d t+p(0) \cos \theta+p^{\prime}(0) \sin \theta .
$$

By differentiating, one has a.e.

$$
\left\{\begin{array}{l}
x^{\prime}(\theta)=-\rho(\theta) \sin \theta \\
y^{\prime}(\theta)=\rho(\theta) \cos \theta
\end{array}\right.
$$

Conversely, a convex body can be characterized by a function $p$ of class $C^{1}, 2 \pi$-periodic and satisfying $p+p^{\prime \prime} \geq 0$ in the distribution sense (that is $\int_{0}^{2 \pi}\left(p+p^{\prime \prime}\right) \phi \geq 0$ for all functions $\phi \geq 0$ in $C_{0}^{\infty}([0,2 \pi])$, the infinitely differentiable functions with compact support in $\left.[0,2 \pi]\right)$. In this case, $p+p^{\prime \prime}$ is a non-negative Radon measure and $p$ is the support function of a strictly convex body. In the present work, we mainly deal with strictly convex bodies whose support functions $p$ are at least of class $C^{1,1}$ (except problem of section 3.4). This is obviously the case if $p$ is a trigonometric polynomial, and also in the case of constant width bodies and rotors, see [4, 5]. Therefore, to ensure convexity of a body $K$ represented by $p$, we assume that (3) holds in the classical sense. 
Remark 2.1. The convexity constraint of $K$ can be equivalently expressed in polar coordinates, see 117. Consider a body $K$ of $\mathbb{R}^{2}$ whose boundary is described by

$$
\left\{\begin{array}{l}
x(\theta)=f(\theta) \cos \theta, \\
y(\theta)=f(\theta) \sin \theta
\end{array}\right.
$$

where $f$ is a periodic continuous function. By standard computations, convexity of $K$ amounts to saying that $u+u^{\prime \prime} \geq 0$, where $u=1 / f$. Notice that the convexity constraint is similar to (3). The parametrization by support function takes easily into account width constraints (see problems of section 3) whereas the polar parametrization handles other constraints.

By standard computations, the area of a convex body $K$ can be expressed by:

$$
A(p)=\frac{1}{2} \int_{0}^{2 \pi}\left(p^{2}(\theta)-p^{\prime 2}(\theta)\right) d \theta=\frac{1}{2} \int_{0}^{2 \pi} p(\theta)\left(p(\theta)+p^{\prime \prime}(\theta)\right) d \theta .
$$

The previous expression is well-defined for a strictly convex body, since then $p$ is $C^{1}$. For a general convex body, the previous expression must be understood as the product of a continuous function and a Radon measure (for example if $K$ is a polygon). The perimeter of $K$ is linear with respect to $p$ :

$$
P(p)=\int_{0}^{2 \pi} p(\theta) d \theta .
$$

The Fourier series of $p$ can be written

$$
p(\theta)=\sum_{k \in \mathbb{Z}} p_{k} e^{i k \theta}
$$

with Fourier coefficients

$$
p_{k}=\frac{1}{2 \pi} \int_{0}^{2 \pi} p(\theta) e^{-i k \theta} d \theta=a_{k}-i b_{k},
$$

satisfying $\bar{p}_{k}=p_{-k}$, so that we can alternatively write

$$
p(\theta)=p_{0}+\sum_{k \geq 1} 2 \operatorname{Re}\left(p_{k} e^{i k \theta}\right)=a_{0}+2 \sum_{k \geq 1}\left(a_{k} \cos k \theta+b_{k} \sin k \theta\right) .
$$

Notice that $p_{0}=a_{0}$. By Parseval equality, the area of $K$ can be expressed as follows:

$$
A(p)=\pi \sum_{k \in \mathbb{Z}}\left(1-k^{2}\right)\left|p_{k}\right|^{2}=\pi a_{0}^{2}+2 \pi \sum_{k \geq 2}\left(1-k^{2}\right)\left(a_{k}^{2}+b_{k}^{2}\right)
$$

and the perimeter of $K$ is obviously $P(p)=a_{0}$. Naturally, $A(p)$ and $P(p)$ do not depend on $p_{1}$ as this coefficient can be chosen zero by translating $K$ along vector $\left(-2 a_{1}, 2 b_{1}\right)$.

\subsection{Convexity constraint as a semidefinite programming problem}

This section is devoted to the reformulation of the convexity constraint into a semidefinite programming (SDP) problem. We first set some notations: 
- Let $z(\theta)=\left(1, e^{i \theta}, \ldots, e^{i m \theta}\right) \in \mathbb{C}^{m+1}, \theta \in \mathbb{R}$.

- Let $0 \leq k \leq m$ and $T_{k} \in \mathbb{R}^{(m+1) \times(m+1)}$ denote the Toeplitz matrix with ones on the $k$-th subdiagonal and zeros elsewhere (lower shift matrix). Similarly define $T_{-k}$ as the transpose of $T_{k}$. Note that $T_{0}$ is the identity matrix.

- $A^{*}$ denotes the transpose conjugate of a matrix $A$.

- The notation $A \succeq 0$ means that matrix $A$ is hermitian positive semidefinite, i.e. with nonnegative real eigenvalues.

- We denote by $A \cdot B$ the inner product of two matrices $A$ and $B$ of the same size, that is the trace of the product of $A^{*}$ by $B$.

Given a hermitian matrix $Q \in \mathbb{C}^{(m+1) \times(m+1)}$, let

$$
q(\theta)=z^{*}(\theta) Q z(\theta)=\sum_{k=-m}^{m} q_{k} e^{i k \theta}
$$

be the value of the quadratic form defined by $Q$ and evaluated at $z(\theta)$, where the star denotes transpose conjugate. Function $q(\theta)$ is a trigonometric polynomial of degree $m$. We next recall a classical lemma showing the linear dependence between entries of matrix $Q$ and Fourier coefficients $q_{k}$ (see [7]).

Lemma 2.1. Hermitian matrix $Q$ and trigonometric polynomial $q(\theta)$ sastify relation (9) if and only if

$$
q_{k}=T_{k} \cdot Q, \quad k=0,1, \ldots, m
$$

Proof. Denoting by $q_{j, k}$ the $(j, k)$-th entry of matrix $Q$, we write:

$$
q(\theta)=\sum_{j=1}^{m+1} q_{j, j}+\sum_{k=1}^{m} \sum_{j=1}^{m+1-k} q_{j, j+k} e^{i k \theta}+\sum_{k=1}^{m} \sum_{j=k+1}^{m+1} q_{j, j-k} e^{-i k \theta}
$$

and as $Q$ is hermitian, we have:

$$
q(\theta)=\sum_{j=1}^{m+1} q_{j, j}+\sum_{k=1}^{m}\left(e^{i k \theta} \sum_{j=1}^{m+1-k} q_{j, j+k}+e^{-i k \theta} \sum_{j=1}^{m+1-k} \bar{q}_{j, j+k}\right) .
$$

We obtain relation (10) by using $\sum_{j=1}^{m+1-k} q_{j, j+k}=\sum_{j=1}^{m+1-k} \bar{q}_{j, j+k}=T_{k} \cdot Q$.

Notice that if $Q$ is real, then $q(\theta)=T_{0} \cdot Q+\sum_{k=1}^{m} 2 \cos (k \theta) T_{k} \cdot Q$ becomes a real trigonometric polynomial.

Let us now consider the set of convex bodies whose support functions are trigonometric polynomials of degree $m$ :

$$
p(\theta)=\sum_{k=-m}^{m} p_{k} e^{i k \theta}
$$


Theorem 2.1. Let $K$ be a convex body with polynomial support function (11). Then there exists a matrix $Q \in \mathbb{C}^{(m+1) \times(m+1)}$ such that

$$
Q \succeq 0, \quad\left(1-k^{2}\right) p_{k}=T_{k} \cdot Q, \quad k=0,1, \ldots, m .
$$

Conversely, any polynomial (11) satisfying relations (12) for some matrix $Q$ is the support function of a convex body $K$.

Proof. Differentiate twice the support function of $K$ to obtain $p(\theta)+p^{\prime \prime}(\theta)=\sum_{k=-m}^{m}(1-$ $\left.k^{2}\right) p_{k} e^{i k \theta}$. Recalling relation (3), function $p+p^{\prime \prime}$ is a non-negative trigonometric polynomial, and the Fejér-Riesz theorem ensures that there exists a trigonometric polynomial $r(\theta)$ such that $p(\theta)+p^{\prime \prime}(\theta)=|r(\theta)|^{2}$ with $r(\theta)=\sum_{k=0}^{m} r_{k} e^{i k \theta}$. It follows that $|r(\theta)|^{2}=\sum_{0 \leq j, k \leq m} r_{j} \bar{r}_{k} e^{i(j-k) \theta}$. Denote by $Q$ the matrix with entries $q_{j, k}=\sum_{0 \leq j, k \leq m} r_{j} \bar{r}_{k}$. By construction, $\bar{Q}$ is positive semidefinite hermitian of size $m+1$, and we can apply Lemma 2.1 on $q(\theta)=|r(\theta)|^{2}$.

Given $p$ in (11), finding a matrix $Q$ satisfying (12) amounts geometrically to finding a point in the intersection of an affine subspace and the convex cone of positive semidefinite matrices. Finding jointly $Q$ and $p$ (presumably satisfying further linear constraints) is equivalent to solving a semidefinite programming (SDP) problem. For more information on the SDP representation of the convex cone of nonnegative trigonometric polynomials, see [14] or [7].

\section{Constant width planar bodies}

In this section we apply the procedure described in the previous section to the minimization of the area for the four problems mentioned in the introduction. We start with convex planar bodies of constant width. If $K \subset \mathbb{R}^{2}$ is a strictly convex body of support function $p$, then the width function is

$$
w(\theta)=p(\theta)+p(\theta+\pi)
$$

and it represents the distance between two different parallel support lines to $K$.

\subsection{Concave SDP problem}

As recalled in [4], any planar constant width body $K$ can be represented by a $2 \pi$-periodic function $p \in C^{1,1}$ which satisfies

$$
\left\{\begin{array}{l}
p(\theta)+p(\theta+\pi)=1, \forall \theta \in \mathbb{R} \\
p+p^{\prime \prime} \geq 0 \text { a.e. }
\end{array}\right.
$$

By taking into account this width constraint, the Fourier series of $p$ becomes

$$
p(\theta)=\frac{1}{2}+2 \operatorname{Re}\left(p_{1} e^{i \theta}\right)+2 \sum_{k \geq 1}\left(a_{2 k+1} \cos (2 k+1) \theta+b_{2 k+1} \sin (2 k+1) \theta\right),
$$

and the area becomes:

$$
A(p)=\frac{\pi}{4}+2 \pi \sum_{k \geq 1}\left(1-(2 k+1)^{2}\right)\left(a_{2 k+1}^{2}+b_{2 k+1}^{2}\right) .
$$


A standard optimization problem consists in determining the constant width convex body of maximal and minimal area. Since any constant width body has the same perimeter as the ball of same diameter (Barbier's theorem), it follows by the isoperimetric inequality that the maximizer is the ball, with area $\frac{\pi}{4} \simeq 0.78540$. It has been proved by Blaschke and Lebesgue in 1920 that the minimizer is the Reuleaux triangle which consists of the intersection of three discs of unit radius centered at the three vertices of an equilateral triangle of unit side, see figure 11. The area of the Reuleaux triangle is equal to $\frac{\pi}{2}-\frac{\sqrt{3}}{2} \simeq 0.70477$. This result has been revisited recently by optimal control theory and calculus of variation [4, 10].
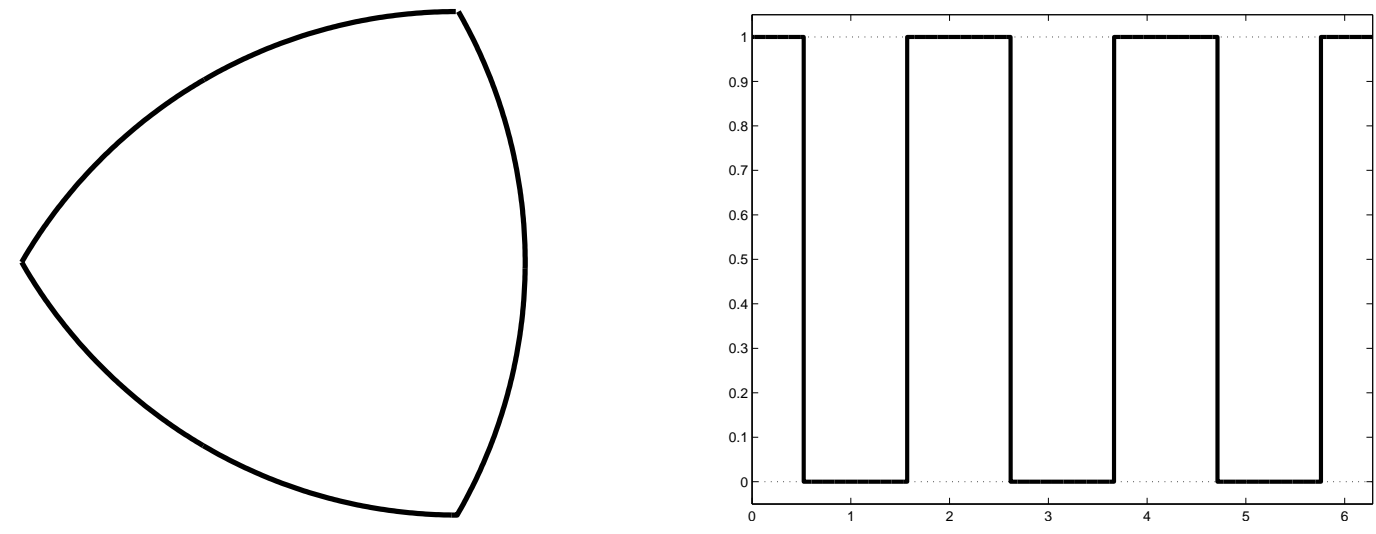

Figure 1: Reuleaux triangle (left) and its radius of curvature (right).

If $p_{R}$ is the Fourier series of the support function of the Reuleaux triangle, the radius of curvature $\rho_{R}$ satisfies for a.e. $\theta \in \mathbb{R}$ :

$$
\rho_{R}(\theta)=p_{R}(\theta)+p_{R}^{\prime \prime}(\theta)=\mathbb{I}_{\left[-\frac{\pi}{6}, \frac{\pi}{6}[\right.}+\mathbb{I}_{\left[\frac{\pi}{2}, \frac{2 \pi}{3}[\right.}+\mathbb{I}_{\left[\pi, \frac{4 \pi}{3}[\right.}=\frac{1}{2}+\frac{2}{\pi} \sum_{k \geq 0} \frac{(-1)^{k}}{2 k+1} \cos (6 k+3) \theta,
$$

and it is therefore lacunary. In the above expression, $\mathbb{I}_{J}$ denotes the indicator function of a set $J$.

Let us now consider the set of trigonometric polynomials $p$ of degree $m=2 N+1$ of the form:

$$
p(\theta)=\frac{1}{2}+2 \sum_{k=0}^{N}\left(a_{2 k+1} \cos (2 k+1) \theta+b_{2 k+1} \sin (2 k+1) \theta\right)
$$

and satisfying (13). The area of the body with support function $p$ is

$$
A(p)=\frac{\pi}{4}+2 \pi \sum_{k=1}^{N}\left(1-(2 k+1)^{2}\right)\left(a_{2 k+1}^{2}+b_{2 k+1}^{2}\right)
$$

and hence the minimization problem we consider is

$$
\begin{array}{ll}
\min _{p} & A(p) \\
\text { s.t. } & \text { (13) and (15). }
\end{array}
$$

By using the reformulation of the convexity constraint into an SDP problem, this optimization problem can be transformed as follows. Consider the set of hermitian matrices $Q$ of size $N=2 m+1$ satisfying the affine equations:

$$
T_{0} \cdot Q=\frac{1}{2}, T_{1} \cdot Q=0, T_{2 k} \cdot Q=0, k=1,2, \ldots, m-1
$$


and let

$$
\mathcal{A}(Q)=\frac{\pi}{4}+2 \pi \sum_{k=1}^{m}\left(1-(2 k+1)^{2}\right)\left(\left(\left(T_{2 k+1}+T_{2 k+1}^{*}\right) \cdot Q\right)^{2}+\left(\left(T_{2 k+1}-T_{2 k+1}^{*}\right) \cdot Q\right)^{2}\right) .
$$

Proposition 3.1. Problem (10) is equivalent to:

$$
\begin{array}{ll}
\min _{Q} & \mathcal{A}(Q) \\
\text { s.t. } & Q \succeq 0 \text { and (17) }
\end{array}
$$

which is a semidefinite programming problem with a concave quadratic objective function and a convex feasible set.

Proof. The affine constraint (17) takes into account the expression of $p$ given by (15). The objective function is a reformulation of the expression (8) of the area with Fourier coefficients $p_{k}=a_{k}-i b_{k}$ such that $a_{k}=\left(T_{2 k+1}+T_{2 k+1}^{*}\right) \cdot Q$ and $b_{k}=\left(T_{2 k+1}-T_{2 k+1}^{*}\right) \cdot Q$. The convexity constraint is then equivalent to the semidefinite constraint $Q \succeq 0$. Finally, concavity of the objective function follows from the negative signs of the quadratic terms in (8).

\subsubsection{Concave semidefinite programming}

To solve problem (18) numerically, we use PENBMI, an implementation of a penalty-augmented Lagrangian method handling nonconvex quadratic semidefinite programming problems [15. As far as we know, this is the only publicly available solver that can deal with such optimization problems. We use YALMIP [18] as a Matlab interface to PENBMI.

PENBMI returns local optima (satisfying first-order optimality conditions) with no guarantee of global optimality, unless the optimization problem is convex. Problem (18) is not convex, and since the objective function is concave we may suspect that there are several local (and maybe global) optima at the boundary of the convex feasible set. To avoid getting trapped into a critical point which is not a global optimum, we run PENBMI with several (typically 10) randomly generated initial points.

PENBMI does not handle complex-valued data, so we rewrite the complex constraint $Q=$ $Q_{R}+i Q_{I} \succeq 0$ as the real constraint

$$
\left[\begin{array}{cc}
Q_{R} & Q_{I} \\
-Q_{I} & Q_{R}
\end{array}\right] \succeq 0
$$

where $Q_{R}=Q_{R}^{*}$ is the symmetric real part of $Q$ and $Q_{I}=-Q_{I}^{*}$ is the skew-symmetric imaginary part of $Q$. Then it follows that $a_{k}=\left(T_{2 k+1}+T_{2 k+1}^{*}\right) \cdot Q=\left(T_{2 k+1}+T_{2 k+1}^{*}\right) \cdot Q_{R}$ and $b_{k}=\left(T_{2 k+1}-T_{2 k+1}^{*}\right) \cdot Q=\left(T_{2 k+1}-T_{2 k+1}^{*}\right) \cdot Q_{I}$.

\subsubsection{Real Fourier coefficients}

We first use PENBMI to solve problem (18) with complex Fourier coefficients (both $a_{k}$ and $b_{k}$ are free, i.e. $Q$ is a complex hermitian matrix) and real Fourier coefficients $\left(b_{k}=0\right.$, i.e. $Q$ is 


\begin{tabular}{l|lll|lll} 
degree & 3 & 5 & 7 & 9 & 11 & 13 \\
\hline complex & 0.73631 & 0.73631 & 0.73631 & 0.71976 & 0.71976 & 0.71976 \\
\hline real & 0.73631 & 0.73631 & 0.73631 & 0.71963 & 0.71963 & 0.71963 \\
\hline \hline degree & 15 & 17 & 19 & 21 & 23 & 25 \\
\hline complex & 0.71347 & 0.71347 & 0.71347 & 0.71045 & 0.71045 & 0.71045 \\
\hline real & 0.71319 & 0.71319 & 0.71319 & 0.71003 & 0.71003 & 0.71003
\end{tabular}

Table 1: Minimum area vs. degree, for complex and real Fourier coefficients.

a real symmetric matrix) for increasing values of the Fourier series truncation degree $m$. Our results are reported in table 1, to 5 significant digits.

First, we see from table 11 that the area does not decrease strictly monotonically: only the harmonics of orders $6 k+3$, with $k$ integer, contribute, which is consistent with the lacunary Fourier series (14).

Second, repeated experiments reveal that the behavior of PENBMI is much more consistent on different runs with random initial conditions when restricting the search to real Fourier coefficients. The algorithm converges almost everytime to the same local minimum, which leads us to conclude that it is actually a global minimum (even though we cannot prove this rigorously). In the case of complex Fourier coefficients, the objective function is invariant upon multiplication of $p_{k}=a_{k}-i b_{k}$ by any complex number of unit magnitude, and hence there are more degrees of freedom. As a result, the algorithm gets more often trapped around suboptimal critical points.

Third, and most importantly, there is no significant contribution provided by the complex parts $b_{k}$ when the degree increases. Notice that in view of (14), it is natural to force this constraint: it is always possible to assume that the radius of curvature $\rho_{R}$ of the Reuleaux triangle is even (by doing a translation). By integration, its support function $p_{R}$ is also even and we can restrict the optimization problem to coefficients $a_{k}$.

For these reasons, we decide to enforce $b_{k}=0$ and to search for a real symmetric matrix $Q$ for orders of the form $6 k+3$, in the experiments below.

\subsubsection{Approximations}

On figure 2 we represent the minimizers and corresponding radii of curvature obtained for various values of the truncation degree $m$, and on table 2 we report the corresponding Fourier coefficients, to 3 significant sigits.

For $N=33$, a typical computational time for one run of PENBMI is less than 5 seconds on a standard PC. 


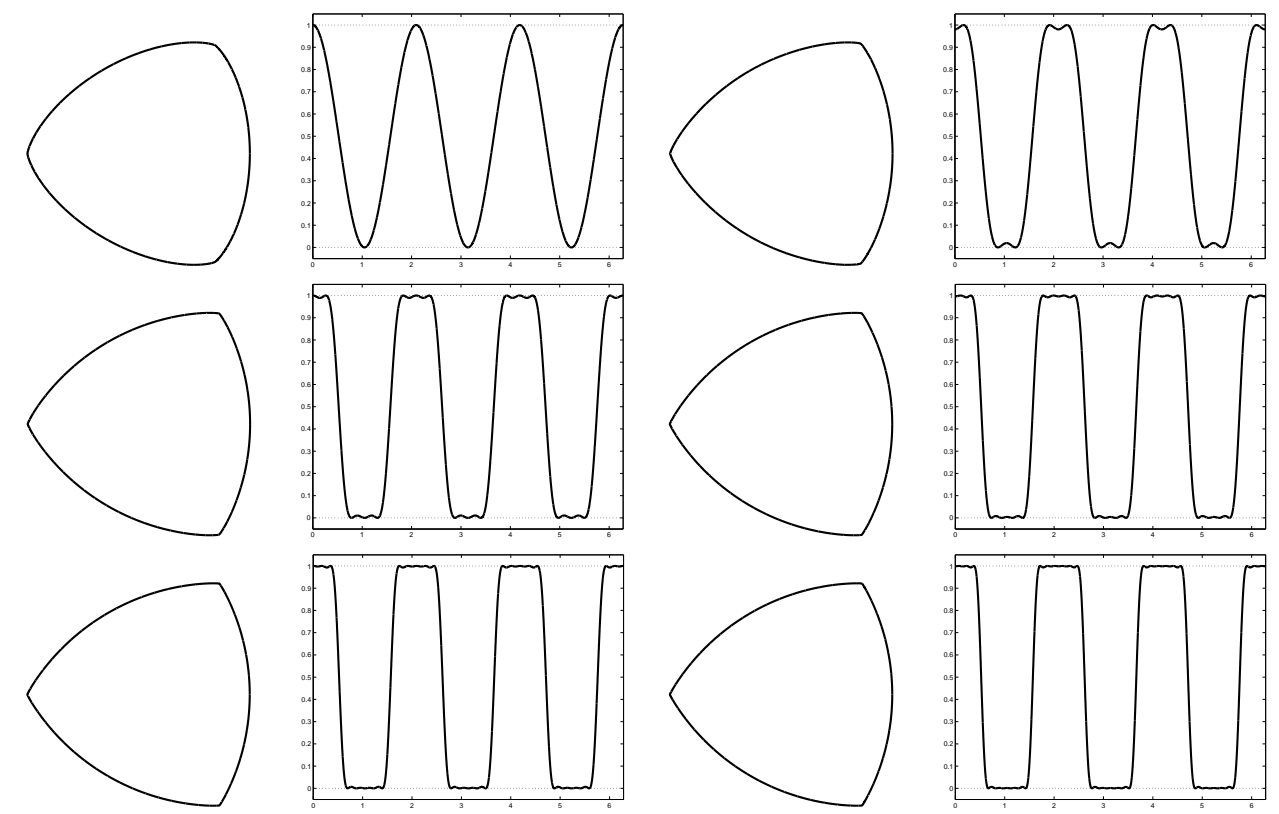

Figure 2: Minimizers and radii of curvature obtained for degrees 3, 9, 15, 21, 27, 33 .

\begin{tabular}{l|l} 
degree & Fourier coefficients \\
\hline \hline 3 & $a_{0}=0.500, a_{3}=-0.0313$ \\
\hline 9 & $a_{0}=0.500, a_{3}=-0.0361, a_{5}=5.5210^{-14}, a_{7}=-1.2310^{-14}, a_{9}=6.0610^{-4}$ \\
\hline 15 & $a_{0}=0.500, a_{3}=-0.0377, a_{5}=9.1510^{-12}, a_{7}=2.8610^{-12}, a_{9}=8.8110^{-4}$, \\
& $a_{11}=-2.3310^{-12}, a_{13}=-1.1310^{-12}, a_{15}=-8.3410^{-5}$ \\
\hline 21 & $a_{0}=0.500, a_{3}=-0.0385, a_{5}=1.0210^{-13}, a_{7}=1.6010^{-14}, a_{9}=1.0210^{-3}$, \\
& $a_{11}=-2.7010^{-14}, a_{13}=-9.5110^{-15}, a_{15}=-1.3910^{-4}, a_{17}=7.0810^{-15}$, \\
& $a_{19}=3.7010^{-15}, a_{21}=2.1110^{-5}$
\end{tabular}

Table 2: Fourier coefficients for degrees 3, 9, 15, 21.

\subsubsection{Low degree bodies}

In the case $m=3$, problem (16) restricted to real coefficients reads

$$
\begin{array}{ll}
\min _{a_{3}} & \frac{\pi}{4}-16 \pi a_{3}^{2} \\
\text { s.t. } & \frac{1}{2}-16 a_{3} \cos 3 \theta \geq 0, \forall \theta \in \mathbb{R}
\end{array}
$$

and from inspection we obtain optimal values $a_{3}= \pm \frac{1}{32}$, support functions $p(\theta)=\frac{1}{2} \pm \frac{1}{16} \cos 3 \theta$ and radii of curvature $\rho(\theta)=\frac{1}{2}(1 \pm \cos 3 \theta)$. This should be compared with the results obtained in [19, Section 3], where the author observes graphically that the support function $p(\theta)=$ $\frac{4}{9}+\frac{1}{9} \cos ^{2} \frac{3}{2} \theta=\frac{1}{2}+\frac{1}{18} \cos 3 \theta$ shapes a smooth convex body of constant width whose boundary is an irreducible algebraic plane curve of degree 8 .

In the case $m=5$, problem (16) restricted to real coefficients reads

$$
\begin{array}{ll}
\min _{a_{3}, a_{5}} & \frac{\pi}{4}-16 \pi a_{3}^{2}-48 \pi a_{5}^{2} \\
\text { s.t. } & \frac{1}{2}-16 a_{3} \cos 3 \theta-48 a_{5} \cos 5 \theta \geq 0, \forall \theta \in \mathbb{R} .
\end{array}
$$

On figure 3 we represent the two-dimensional convex feasible set. Numerically, we observe that the minimum area is achieved at the boundary points $a_{3}= \pm \frac{1}{32}$ on the axis $a_{5}=0$. 


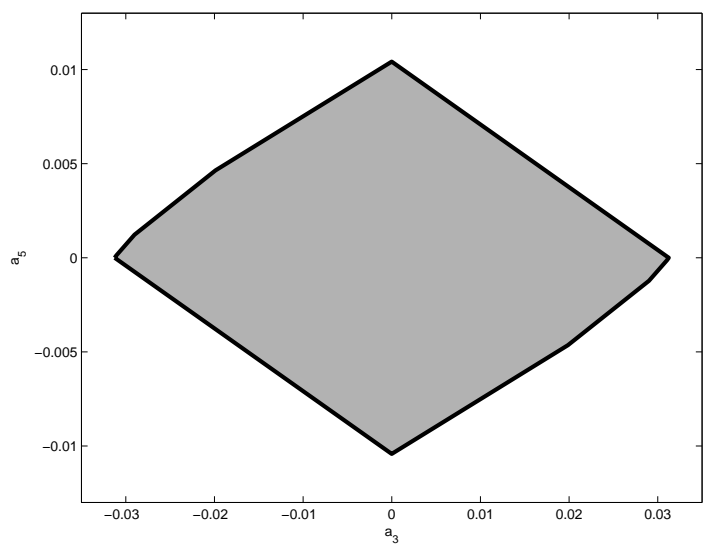

Figure 3: In shaded gray, admissible Fourier coefficients for constant width convex bodies with trigonometric polynomial support functions of fifth degree.

In the case $m=7$, problem (16) restricted to real coefficients reads

$$
\begin{array}{ll}
\min _{a_{3}, a_{5}, a_{7}} & \frac{\pi}{4}-16 \pi a_{3}^{2}-48 \pi a_{5}^{2}-96 \pi a_{7}^{2} \\
\text { s.t. } & \frac{1}{2}-16 a_{3} \cos 3 \theta-48 a_{5} \cos 5 \theta-96 a_{7} \cos 7 \theta \geq 0, \forall \theta \in \mathbb{R} .
\end{array}
$$

On figure 4 we represent the three-dimensional convex feasible set. Numerically, we observe that the minimum area is achieved at the boundary points $a_{3}= \pm \frac{1}{32}$ on the plane $a_{5}=a_{7}=0$.
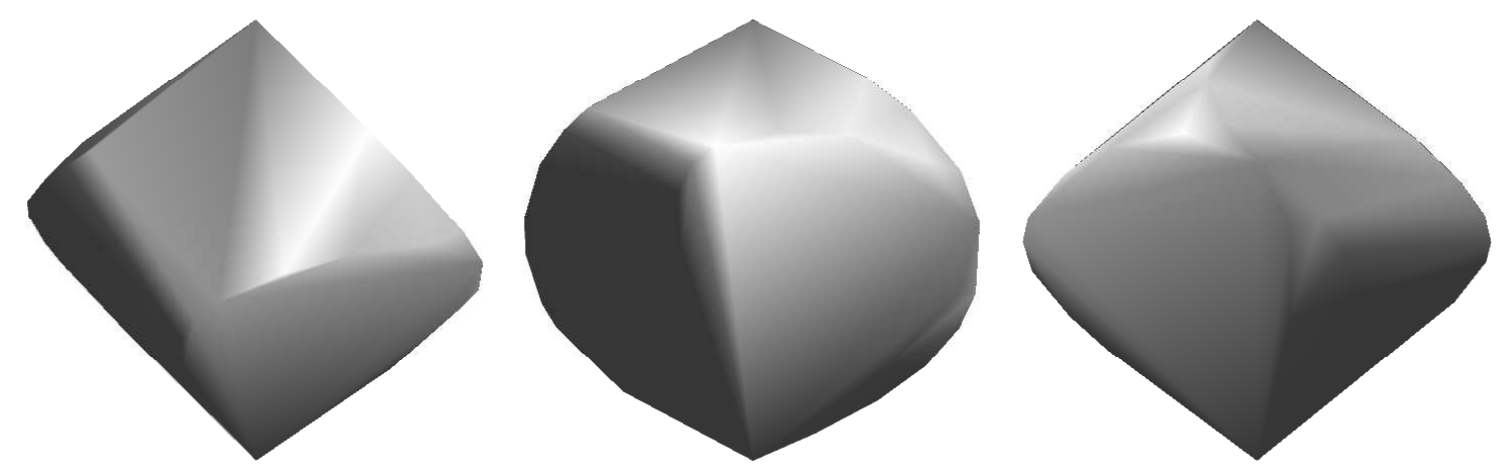

Figure 4: Three different views of the set of admissible Fourier coefficients for constant width convex bodies with trigonometric polynomial support functions of seventh degree.

\subsection{Planar rotors}

A rotor is a generalization of a constant width body of $\mathbb{R}^{2}$ in an $n$-gon (that is a regular polygon with $n$ sides). Let $P$ a polygon with $n$ sides. We say that a regular polygon $P$ with $n$ sides is tangential to a convex body $K$ if $K \subset P$ and every side of $P$ has a non-empty intersection with $K$. Geometrically speaking, a rotor $K$ in an $n$-gon is a convex body such that each tangential regular polygon $P$ has the same perimeter. It can be proved [4, 10] that a convex body $K$ is a rotor of an $n$-gon if and only if its support function $p$ is a $2 \pi$-periodic 
function of class $C^{1,1}$ such that:

$$
\left\{\begin{array}{l}
p(\theta)-2 \cos \delta p(\theta+\delta)+p(\theta+2 \delta)=1, \forall \theta \in \mathbb{R} \\
p+p^{\prime \prime} \geq 0 \text { a.e. }
\end{array}\right.
$$

where $\delta=\frac{2 \pi}{n}$. The radius $r$ of the inscribed circle to a tangential polygon $P$ of a rotor $K$ satisfies $4 r \sin ^{2}\left(\frac{\pi}{n}\right)=1$. In the case $n=4$, relations (19) yield relations (13) and $r=\frac{1}{2}$, corresponding to a body of constant unit width, studied in the previous section. The Fourier series of the support function of a rotor is:

$$
p(\theta)=r+\sum_{k \in J} p_{k} e^{i k \theta}
$$

where $J=(n \mathbb{Z}+1) \cup(n \mathbb{Z}-1)$, see [4]. Equivalently, we have:

$$
\begin{aligned}
p(\theta)=r+2 \operatorname{Re}\left(p_{1} e^{i \theta}\right) & +2 \sum_{k \geq 1}\left(a_{k n+1} \cos (k n+1) \theta+b_{k n+1} \sin (k n+1) \theta\right) \\
& +2 \sum_{k \geq 1}\left(a_{k n-1} \cos (k n-1) \theta+b_{k n-1} \sin (k n-1) \theta\right) .
\end{aligned}
$$

It has been proved that the rotor of minimal area is the regular-trammel rotor described by Goldberg [4, 9]. The minimizers for $n=3$ and $n=5$ and the corresponding radii of curvature are represented on figure 5. When $n=3$ the minimum area is $\frac{\pi}{3}-\frac{\sqrt{3}}{2} \simeq 0.18117$. When $n=5$ the minimum area is $\frac{\pi}{16 \sin (\pi / 5)^{4}}-\frac{10(\cot (3 \pi / 20)-1)-\pi\left(1+\cot (\pi / 5)^{2}\right)}{32 \sin (\pi / 5)^{2} \cos (\pi / 5)^{2}} \simeq 1.5713$. The exact value of the minimum area for any regular rotor can be found in [4] via Fourier series.
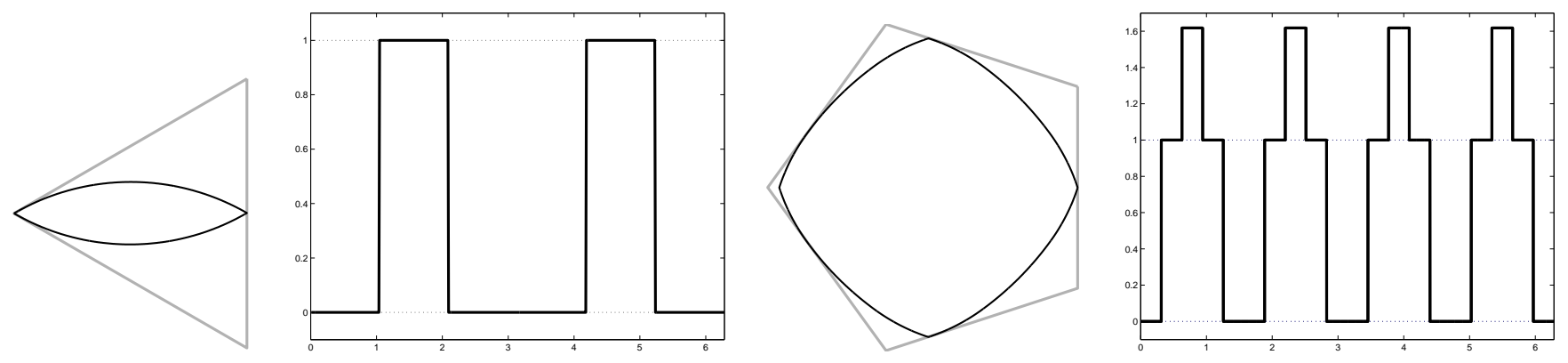

Figure 5: Optimal rotors (black) inscribed in polygons (gray) and radii of curvature for $n=3$ (left) and $n=5$ (right).

Following the same scheme as in the case of constant width bodies, we now consider the set of trigonometric polynomials $p$ of degree $m=N n+1$ which satisfy (19) and are given by:

$$
\begin{aligned}
p(\theta)=r+2 \operatorname{Re}\left(p_{1} e^{i \theta}\right)+ & 2 \sum_{k=1}^{N}\left(a_{k n+1} \cos (k n+1) \theta+b_{k n+1} \sin (k n+1) \theta\right) \\
& +2 \sum_{k=1}^{N}\left(a_{k n-1} \cos (k n-1) \theta+b_{k n-1} \sin (k n-1) \theta\right) .
\end{aligned}
$$

The area of a rotor is

$$
A(p)=\pi r^{2}+2 \pi \sum_{k=1}^{N}\left(1-(k n+1)^{2}\right)\left(a_{k n+1}^{2}+b_{k n+1}^{2}\right)+\left(1-(k n-1)^{2}\right)\left(a_{k n-1}^{2}+b_{k n-1}^{2}\right)
$$



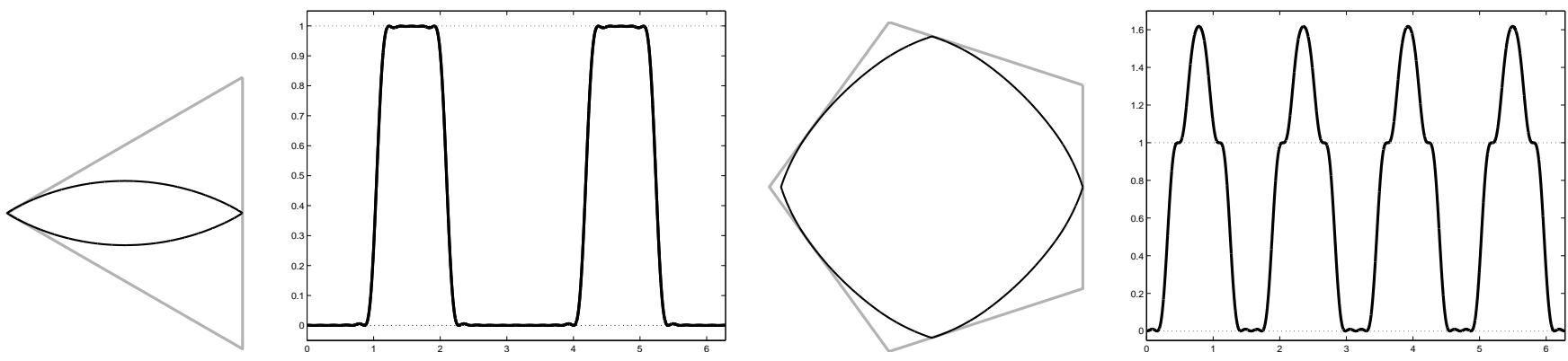

Figure 6: Optimal rotors (black) inscribed in polygons (gray) and radii of curvature of degree 30 for $n=3$ (left) and $n=5$ (right).

and finding the rotor of minimal area can be then stated as follows:

$$
\min _{p} A(p)
$$

s.t. $\quad(19)$ and $(20)$.

The convex bodies obtained by this procedure for $m=30$ are represented on figure 6. Tables 3 and 1 report the areas of the rotors obtained by this procedure for $n=3$ and $n=5$, respectively. The coordinates of the $n$ vertices of the tangential polygon to a rotor $K$ can be computed by the formula $(0 \leq k \leq n-1)$ :

$$
\left\{\begin{array}{l}
x_{k}=\frac{1}{\sin \delta}(p(k \delta) \sin (k+1) \delta-p((k+1) \delta) \sin k \delta), \\
y_{k}=\frac{1}{\sin \delta}(p((k+1) \delta) \cos k \delta-p(k \delta) \cos (k+1) \delta) .
\end{array}\right.
$$

It is obtained by intersecting the $n$ support lines of equation $x \cos k \delta+y \sin k \delta=p(k \delta)$ (see [3], [4]).

\begin{tabular}{c|cccccc} 
degree & 1 & $2-3$ & $4-7$ & $8-9$ & $10-13$ & $14-15$ \\
\hline area & 0.33333 & 0.29089 & 0.22973 & 0.21416 & 0.20129 & 0.19699 \\
\hline \hline degree & $16-19$ & $20-21$ & $22-25$ & $26-28$ & $29-32$ & \\
\hline area & 0.19205 & 0.19007 & 0.18797 & 0.18696 & 0.18582 &
\end{tabular}

Table 3: Optimal rotor areas for $n=3$.

\begin{tabular}{c|ccccc} 
degree & $1-3$ & $4-13$ & $14-15$ & $16-23$ & $24-35$ \\
\hline area & 1.6450 & 1.5901 & 1.5865 & 1.5807 & 1.5763
\end{tabular}

Table 4: Optimal rotor areas for $n=5$.

\subsection{Constant width bodies of revolution}

It is standard that any convex boby $K$ obtained by rotation of a constant width planar body $K_{0}$ around one of its axis a symmetry $\Delta$ can be represented by a $2 \pi$-periodic support function 
$p$ of class $C^{1,1}$ that satisfies

$$
\left\{\begin{array}{l}
p(\theta)+p(\theta+\pi)=1, \forall \theta \in \mathbb{R} \\
p(\theta)=p(\pi-\theta), \forall \theta \in \mathbb{R} \\
p+p^{\prime \prime} \geq 0 \text { a.e. }
\end{array}\right.
$$

More details on this parametrization and on the following computations can be found in [3], 13. Without loss of generality, if $\Delta$ is the $z$-axis, then the boundary of $K$ is parametrized by

$$
\left\{\begin{array}{l}
x(\theta, \varphi)=\left(p(\theta) \cos \theta-p^{\prime}(\theta) \sin \theta\right) \cos \varphi \\
y(\theta, \varphi)=\left(p(\theta) \cos \theta-p^{\prime}(\theta) \sin \theta\right) \sin \varphi \\
z(\theta, \varphi)=p(\theta) \sin \theta+p^{\prime}(\theta) \cos \theta
\end{array}\right.
$$

with $\theta \in[0,2 \pi], \varphi \in[0, \pi]$. Notice that any function $p$ satisfying (21) must be such that $p(0)=\frac{1}{2}, p^{\prime}\left(\frac{\pi}{2}\right)=0$ and

$$
\int_{-\frac{\pi}{2}}^{\frac{\pi}{2}} p(\theta) \cos \theta d \theta=1
$$

Indeed, one has $\int_{-\frac{\pi}{2}}^{\frac{\pi}{2}} p(\theta) \cos \theta d \theta=\int_{0}^{\frac{\pi}{2}} p(\theta) \cos \theta d \theta+\int_{-\frac{\pi}{2}}^{0} p(\theta) \cos \theta d \theta$, and $\int_{-\frac{\pi}{2}}^{0} p(\theta) \cos \theta d \theta=$ $-\int_{\frac{\pi}{2}}^{\pi}(1-p(\theta)) \cos \theta d \theta=1-\int_{0}^{\frac{\pi}{2}} p(\theta) \cos \theta d \theta$, which gives (23). The surface of revolution $S(p)$ of $K$ is then given by:

$$
S(p)=2 \pi \int_{-\frac{\pi}{2}}^{\frac{\pi}{2}}\left(p(\theta)+p^{\prime \prime}(\theta)\right)\left(p(\theta) \cos \theta-p^{\prime}(\theta) \sin \theta\right) d \theta=2 \pi \int_{-\frac{\pi}{2}}^{\frac{\pi}{2}}\left(p^{2}(\theta)-\frac{1}{2} p^{2}(\theta)\right) \cos \theta d \theta
$$

see [3]. Using (21), the previous expression can be written on $\left[0, \frac{\pi}{2}\right]$ as follows:

$$
S(p)=4 \pi \int_{0}^{\frac{\pi}{2}}\left(p^{2}(\theta)-p^{2}(\theta)\right) \cos \theta d \theta+2 \pi-4 \pi \int_{0}^{\frac{\pi}{2}} p(\theta) \cos \theta d \theta .
$$

Therefore, for the ball of radius $\frac{1}{2}, p(\theta)=\frac{1}{2}$ and $S(p)=\pi$, whereas for the rotated Reuleaux triangle, the support function on $\left[0, \frac{\pi}{2}\right]$ is:

$$
\left\{\begin{array}{l}
p(\theta)=\frac{1}{2} \cos \theta, \theta \in\left[0, \frac{\pi}{3}\right] \\
p(\theta)=1+\frac{1}{2} \cos \theta-\cos \left(\theta-\frac{\pi}{3}\right), \theta \in\left[\frac{\pi}{3}, \frac{\pi}{2}\right]
\end{array}\right.
$$

and $S(p)=2 \pi-\frac{\pi^{2}}{3} \approx 2.9933$

Using (21), the decomposition of $p$ into Fourier series writes:

$$
p(\theta)=\frac{1}{2}+2 \sum_{k \geq 0} b_{2 k+1} \sin (2 k+1) \theta .
$$

The surface of revolution can be then rewritten in terms of Fourier coefficients of $p$. Consider for $j, k \geq 0$ the real:

$$
\nu_{j k}=\frac{32(-1)^{j+k} j k(1+j+k+j k)}{(2 j+2 k+3)(2 j+2 k+1)(2 j-2 k+1)(2 j-2 k-1)} .
$$


Proposition 3.2. If $K$ is a constant width body of revolution obtained by rotation of $K_{0}$, and $p$ is the support function of $K_{0}$, then the surface of $K$ is:

$$
S(p)=\pi+8 \pi \sum_{j, k \geq 0} \nu_{j k} b_{2 j+1} b_{2 k+1} .
$$

Proof. Let $q=p-\frac{1}{2}$ and $\alpha_{j}=2 b_{2 j+1}, j \geq 0$. From (24), we have:

$$
S(q)=2 \pi\left(\int_{-\frac{\pi}{2}}^{\frac{\pi}{2}}\left(q^{2}(\theta)-\frac{1}{2} q^{\prime 2}(\theta)\right) \cos \theta d \theta+\int_{-\frac{\pi}{2}}^{\frac{\pi}{2}} p(\theta) \cos \theta d \theta-\frac{1}{4} \int_{-\frac{\pi}{2}}^{\frac{\pi}{2}} \cos \theta d \theta\right) .
$$

A straightforward computation shows that:

$$
\left\{\begin{array}{l}
\int_{-\frac{\pi}{2}}^{\frac{\pi}{2}} q^{2}(\theta) \cos \theta d \theta=\sum_{j, k \geq 0} \lambda_{j, k} \alpha_{2 j+1} \alpha_{2 k+1} \\
\int_{-\frac{\pi}{2}}^{\frac{\pi}{2}} q^{\prime 2}(\theta) \cos \theta d \theta=\sum_{j, k \geq 0}(2 j+1)(2 k+1) \mu_{j, k} \alpha_{2 j+1} \alpha_{2 k+1}
\end{array}\right.
$$

with

$$
\lambda_{j, k}=-\frac{2(-1)^{j+k}\left((2 j+1)^{2}+(2 k+1)^{2}-1\right)}{(2 j+2 k+3)(2 j+2 k+1)(2 j-2 k+1)(2 j-2 k-1)},
$$

and

$$
\mu_{j, k}=-\frac{4(-1)^{j+k}(4 j k+2 j+2 k+1)}{(2 j+2 k+3)(2 j+2 k+1)(2 j-2 k+1)(2 j-2 k-1)} .
$$

Next we can verify that $\nu_{j k}=\lambda_{j k}-\frac{(2 j+1)(2 k+1)}{2} \mu_{j k}$ and that $\int_{-\frac{\pi}{2}}^{\frac{\pi}{2}} p(\theta) \cos \theta d \theta-\frac{1}{4} \int_{-\frac{\pi}{2}}^{\frac{\pi}{2}} \cos \theta d \theta=$ $1 / 2$ by using (23). Relation (26) follows readily.

Let us now state the optimization problem that we consider in the case of constant width bodies of revolution. Consider the set of trigonometric polynomials $p$ of degree $m=2 N+1$ which satisfy (21) and whose Fourier series is given by:

$$
p(\theta)=\frac{1}{2}+2 \sum_{k=0}^{N} b_{2 k+1} \sin (2 k+1) \theta
$$

The surface of the body is

$$
S(q)=\pi+8 \pi \sum_{j, k=0}^{N} \nu_{j k} b_{2 j+1} b_{2 k+1}
$$

and hence the optimization problem we consider is

$$
\begin{array}{ll}
\min _{p} & S(p) \\
\text { s.t. } & \text { 21) and (27). }
\end{array}
$$

The convex body of revolution obtained by this procedure for $m=49$ is represented on figure 7. The optimal surfaces for various degrees are reported in table 8 . 

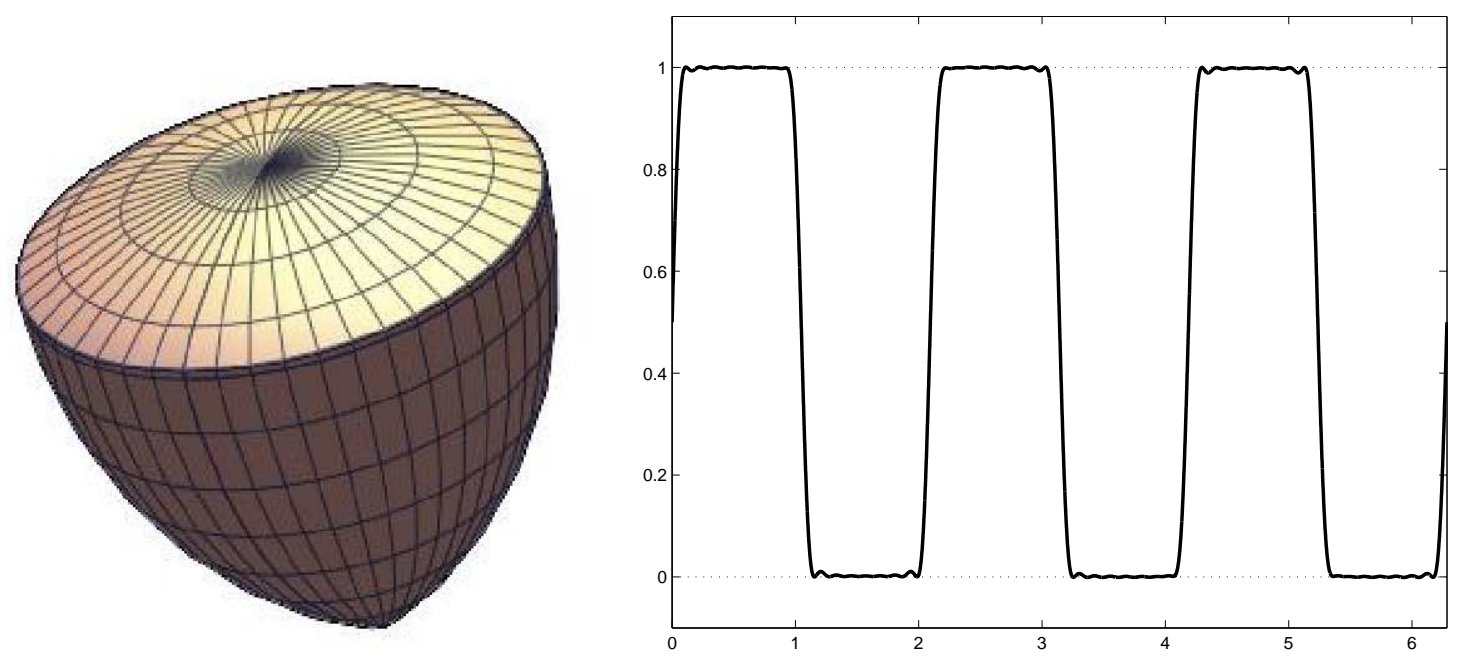

Figure 7: Convex body of revolution and radius of curvature of degree 39.

\begin{tabular}{c|ccccccc} 
degree & $3-5$ & 7 & $9-11$ & 13 & $15-17$ & 19 & $21-23$ \\
\hline surface & 3.0518 & 3.0512 & 3.0218 & 3.0215 & 3.0105 & 3.0102 & 3.0050 \\
\hline \hline degree & 25 & $27-29$ & 31 & $33-35$ & 37 & 39 & \\
\hline surface & 3.0049 & 3.0020 & 3.0019 & 3.0001 & 3.0000 & 2.9989 &
\end{tabular}

Figure 8: Optimal surfaces of constant width convex bodies of revolution.

\subsection{Optimization problem with a relaxation of the width constraint}

In this section, we consider the problem of minimizing the area in a class of convex bodies for which the width lies within two values $w_{1}$ and $w_{2}$ with $w_{1}<w_{2}$. It seems a natural extension of the concept of constant width body. A set satisfying such an inequality constraint is not necessarily strictly convex, and its support function is not of class $C^{1}$, but Lipschitz continuous (see [11]). Let us note $C^{1,0}$ the set of all Lipschitz continuous functions which are $2 \pi$-periodic. In this section, we consider the subset $F$ of $C^{1,0}$ of all functions $p$ satisfying:

$$
\left\{\begin{array}{l}
w_{1} \leq p(\theta)+p(\theta+\pi) \leq w_{2}, \forall \theta \in \mathbb{R} \\
\exists \theta_{1} \in[0,2 \pi], p\left(\theta_{1}\right)+p\left(\theta_{1}+\pi\right)=w_{1}, \\
\exists \theta_{2} \in[0,2 \pi], p\left(\theta_{2}\right)+p\left(\theta_{2}+\pi\right)=w_{2} \\
p+p^{\prime \prime} \geq 0, \text { a.e. }
\end{array}\right.
$$

The function $p$ is the support function of a convex body $K$ for which the width function $w(\theta)=p(\theta)+p(\theta+\pi)$ takes values within $I=\left[w_{1}, w_{2}\right]$, and the two extremal values of $I$ are achieved. Notice that the set $F$ does not define a convex subseet of $C^{1,0}$. Consider the set of trigonometric polynomials $p$ of degree $m \leq N$ :

$$
p(\theta)=p_{0}+2 \sum_{k=1}^{N}\left(a_{k} \cos (k \theta)+b_{k} \sin (k \theta)\right),
$$

and which satisfy (29). If $\theta_{1}$ and $\theta_{2}$ are fixed, the two pointwise constraints of (29) are linear with respect to the Fourier coefficients of $p$. The convexity constraint can be then reformulated as above as a semidefinite constraint. The two inequality constraints on the width function 
are nonnegativity constraints on trigonometric polynomials, and they can also be transformed into semidefinite constraints.

Our goal is to study the optimization problem:

$$
\begin{array}{ll}
\min _{p} & J(p) \\
\text { s.t. } & (29) \text { and }(30)
\end{array}
$$

where $J(p)$ represents plus or minus the area or perimeter of a convex body $K$ whose support function is $p$.

We successively consider the next four problems:

- (P1): maximization of the perimeter s.t. (29) (linear objective)

- (P2): minimization of the perimeter s.t. (29) (linear objective)

- (P3): maximization of the area s.t. (29) (convex quadratic objective)

- (P4): minimization of the area s.t. (29) (concave quadratic objective)

As far as we know, these problems have been studied only in [1, 2]. The authors study (P1), (P2) and (P3) and describe convex bodies solving these problems. The optimality is proved by computing the support function associated to each convex body and by proving that the Hamilton-Jacobi equation is satisfied (sufficient conditions for optimality).

This section aims at finding numerically the optimal shape described in [1, 2]. From the numerical experiments made for (P4), we are able to make a conjecture concerning a solution of this problem.

It is always possible to let $\theta_{1}=0$ (for which the minimal width $w_{1}$ is achieved) by performing a rotation. The value of $\theta_{2}$ (for which the maximal width $w_{2}$ is achieved) cannot be fixed and we currently do not know how to handle this in the optimization procedure. Here we consider the simpler instance consisting in letting $\theta_{2}=\frac{\pi}{2}$, and we take $w_{1}=1, w_{2}=2$.

\subsubsection{Maximization of the perimeter}

For problem (P1), the result proved in [1, 2] is the following. A maximizer of the perimeter under the additional constraint (29) is a convex body $K$ represented by its support function $p$ such that there exists $0<\alpha<\pi$ satisfying

$$
\left\{\begin{array}{l}
p(0)+p(\pi)=w_{1} \\
p(\theta)+p(\theta+\pi)=w_{2}, \theta \in[\alpha, \pi-\alpha] \\
p(\theta)+p^{\prime \prime}(\theta)=p(\theta+\pi)+p^{\prime \prime}(\theta+\pi)=0, \theta \in[0,2 \pi] \backslash[\alpha, \pi-\alpha] .
\end{array}\right.
$$

In the direction $\theta=0$, the width of $K$ is $w_{1}$, whereas for $\theta \in[\alpha, \pi-\alpha]$, the width of $K$ is $w_{2}$. Geometrically speaking, the boundary of $K, \partial K$, has four corners (when $\left.p(\theta)+p^{\prime \prime}(\theta)=0\right)$. Moreover, $\left\{\theta \in[0,2 \pi] \mid w(\theta)=w_{1}\right\}$ is a singleton, hence $\partial K$ is reduced to a line-segment in 
the direction $\theta=0$ and $\theta=\pi$. The boundary of $K$ therefore contains strictly convex parts (when $\left.w(\theta)=w_{2}\right)$ and line-segment $(\theta=0, \pi)$. There is no unicity of such a convex body as $p$ is not uniquely defined by the equation above.

From a numerical point of view, these properties are confirmed (see figure 3.4.1). The radius of curvature obtained numerically is close to a Dirac measure for $\theta=0, \pi$ (corresponding to a line-segment) and is close to zero in four disjoint intervals (corresponding to four corners).
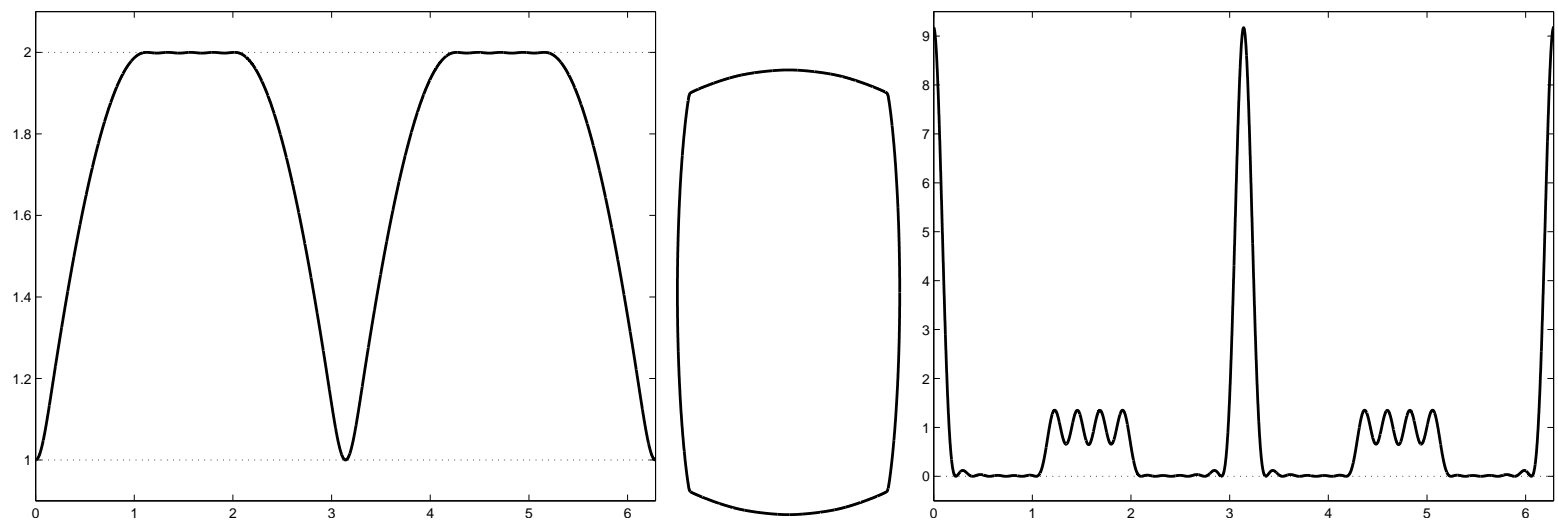

Figure 9: Numerical solution of the perimeter maximization problem for degree 30: width, body and curvature.

\begin{tabular}{c|cccccccc} 
degree & 3 & $4-5$ & $6-7$ & $8-9$ & $10-11$ & $12-13$ & $14-15$ & \\
\hline perimeter & 0.75000 & 0.79635 & 0.80357 & 0.82146 & 0.83386 & 0.83640 & 0.84732 & \\
\hline \hline degree & $16-17$ & $18-19$ & $20-21$ & $22-23$ & $24-25$ & $26-27$ & $28-29$ & 30 \\
\hline perimeter & 0.84906 & 0.85364 & 0.85619 & 0.85756 & 0.86015 & 0.86164 & 0.86265 & 0.86455
\end{tabular}

Figure 10: Numerical solution of the perimeter maximization problem.

\subsubsection{Minimization of the perimeter}

For problem (P2), the result proved in [1, 2] is the following. A minimizer of the perimeter under the additional constraint (29) is a convex body represented by a support function $p$ such that there exists $0<\alpha<\pi$ satisfying

$$
\left\{\begin{array}{l}
p(0)+p(\pi)=w_{2}, \\
p(\theta)+p(\theta+\pi)=w_{1}, \theta \in[\alpha, \pi-\alpha] \\
p(\theta)+p^{\prime \prime}(\theta)=p(\theta+\pi)+p^{\prime \prime}(\theta+\pi)=0, \theta \in[0,2 \pi] \backslash[\alpha, \pi-\alpha] .
\end{array}\right.
$$

Notice that (P1) and (P2) are analogous as the perimeter is linear with respect Fourier coefficients. It is therefore natural to obtain a solution of (P2) by permutation of $w_{1}$ and $w_{2}$. As in section 3.4.1, the numerical results confirm the result obtained in [1, 2]. 

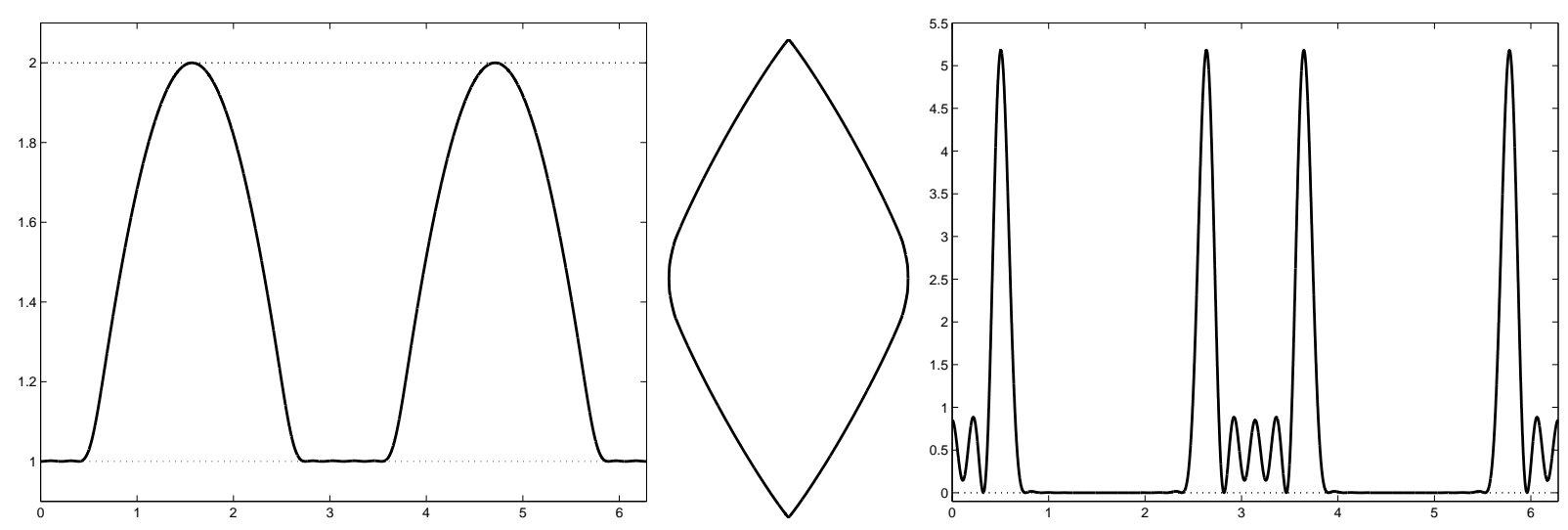

Figure 11: Numerical solution of the perimeter minimization problem for degree 30: width, body and curvature.

\begin{tabular}{c|ccccccc} 
degree & $3-5$ & $6-7$ & $8-9$ & $10-11$ & $12-13$ & $14-15$ & $16-17$ \\
\hline perimeter & 0.75000 & 0.74002 & 0.72951 & 0.72919 & 0.72707 & 0.72370 & 0.72170 \\
\hline \hline degree & $18-19$ & $20-21$ & $22-23$ & $24-25$ & $26-27$ & $28-29$ & 30 \\
\hline perimeter & 0.72157 & 0.72119 & 0.72037 & 0.72022 & 0.72009 & 0.71963 & 0.71936
\end{tabular}

Figure 12: Numerical solution of the perimeter minimization problem.

\subsubsection{Maximization of the area}

For problem (P3), the result proved in [1, 2] is the following. A solution of (P3) is the intersection of a disc of diameter $w_{2}$ and the domain inside two parallel lines symmetric with respect to the center of the disc and at a distance $w_{1}$. Again, the numerical results (see Figure 3.4.3) confirm the theoretical result. Notice the analogy between the numerical solution obtained in 3.4.1 and 3.4.3 (the choice of $\theta_{2}=\pi / 2$ may produce similar numerical solutions, although theoretical maximizers are different).
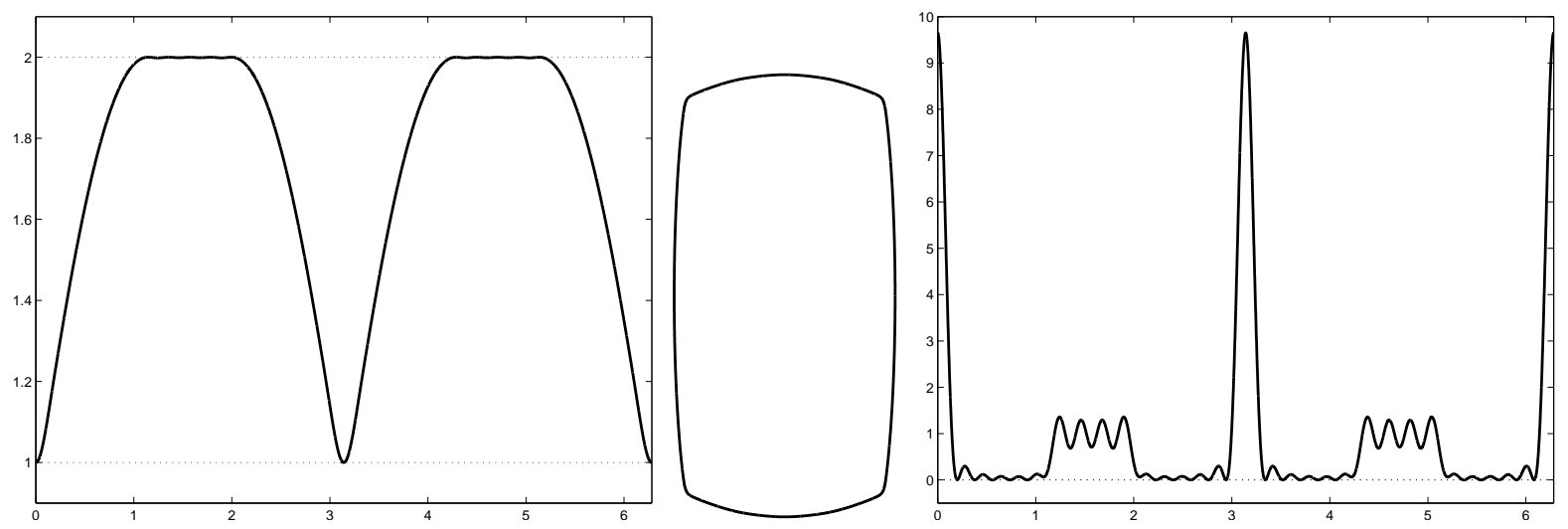

Figure 13: Numerical solution of the area maximization problem for degree 30: width, body and curvature. 


\begin{tabular}{c|cccccccc} 
degree & 3 & $4-5$ & $6-7$ & $8-9$ & $10-11$ & $12-13$ & $14-15$ & $16-17$ \\
\hline area & 1.4726 & 1.6472 & 1.6815 & 1.7388 & 1.7718 & 1.7844 & 1.8098 & 1.8173 \\
\hline \hline degree & $18-19$ & $20-21$ & $22-23$ & $24-25$ & $26-27$ & $28-29$ & 30 & \\
\hline area & 1.8273 & 1.8364 & 1.8411 & 1.8467 & 1.8524 & 1.8552 & 1.8595 &
\end{tabular}

Figure 14: Numerical solution of the area maximization problem.

\subsubsection{Minimization of the area}

For problem (P4), we expect to find a similar shape as for (P3), although (P3) is convex whereas (P4) is not convex due to the concave quadratic objective function. The numerical computations are depicted on Figure 3.4.4.
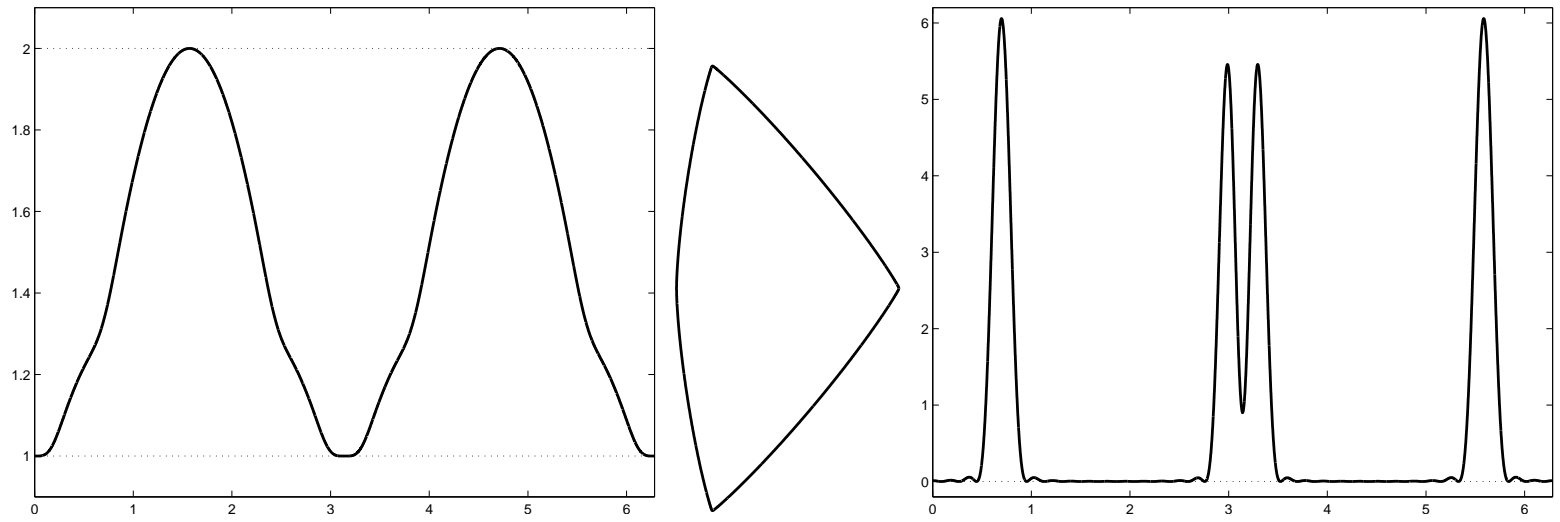

Figure 15: Numerical solution of the area minimization problem for degree 30: width, body and curvature.

\begin{tabular}{c|ccccccc} 
degree & $3-4$ & 5 & 6 & 7 & $8-9$ & 10 & 11 \\
\hline area & 1.4726 & 1.4081 & 1.3937 & 1.3349 & 1.3091 & $1.3060^{*}$ & 1.2563 \\
\hline \hline degree & 12 & 13 & 14 & 15 & 16 & 17 & 18 \\
\hline area & 1.2562 & 1.2319 & 1.2243 & $1.2299^{*}$ & $1.2246^{*}$ & $1.1978^{*}$ & 1.1966 \\
\hline \hline degree & 19 & 20 & 21 & 22 & 23 & 24 & 25 \\
\hline area & 1.1936 & $1.1783^{*}$ & $1.1659^{*}$ & 1.1636 & $1.1484^{*}$ & $1.1640^{*}$ & $1.1465^{*}$ \\
\hline \hline degree & 26 & 27 & 28 & 29 & 30 & & \\
\hline area & 1.1325 & 1.1319 & $1.1353^{*}$ & $1.1281^{*}$ & $1.1271^{*}$ & &
\end{tabular}

Figure 16: Numerical solution of the area minimization problem. A star means that PENBMI cannot guarantee convergence.

\subsubsection{Comments on (P1), (P2), (P3), (P4)}

From a theoretical point of view, both problems (P1), (P2) are linear with respect to $p$. The constraints given by (29) can be decomposed into a convex set $\mathcal{C}_{1}$ (defined by $p+p^{\prime \prime} \geq 0$, $w_{1} \leq w \leq w_{2}$ ) and a non-convex set $\mathcal{C}_{2}$ (defined by $\left.\min _{\theta} w(\theta)=w_{1}, \max _{\theta} w(\theta)=w_{2}\right)$. It is standard that a minimizer $p$ (or a maximizer) of the perimeter cannot take values in the 
interior of $\mathcal{C}_{1}$ on a set of strictly positive measure. It can be then expected that a minimizer $p$ will be such that for a.e. $\theta \in[0,2 \pi], p(\theta)+p^{\prime \prime}(\theta)=0$ or $w(\theta) \in\left\{w_{1}, w_{2}\right\}$. Moreover, for the minimization problem, the set $\left\{\theta \in[0,2 \pi] \mid w(\theta)=w_{2}\right\}$ must be of zero measure. We can therefore expect that the boundary of a minimizer consists of line-segments (separated by $w_{2}$ ) and strictly convex parts such that $w(\theta)=w_{1}$. As the width function $w$ is continuous, there exists a certain interval on which $p(\theta)+p^{\prime \prime}(\theta)=0$ (corresponding to a corner) connecting a line-segment and a strictly convex part. The numerical experiments performed in the previous section confirm these remarks (both for (P1) and (P2).

Similar remarks can be made for (P3) and (P4). First, notice that for (P3), the functional is convex. If the constraint $\min _{\theta} w(\theta)=w_{1}$ is removed, the disc of diameter $w_{2}$ would solve (P3) by the isoperimetric inequality. It can be then expected that the boundary of a maximizer contains arcs of circle of radius $w_{2} / 2$ and also line-segments deparated by $w_{1}$ to ensure the constraint $\min _{\theta} w(\theta)=w_{1}$. Problem (P4) is concave and is more tedious to study. From the numerical computations, we conjecture that a minimizer is a convex polygon with at most four sides.

\section{Convergence of the method}

This section is devoted to the study of the convergence of the method which has been previously discussed. We investigate the case of planar constant width bodies (section 3.1). The proof can be transposed to the case of rotors (see remark 4.2).

In the following, $H^{1}$ denotes the set of functions $p$ which are $2 \pi$-periodic and such that $p_{\mid[0,2 \pi]} \in H^{1}([0,2 \pi])$. The Fourier coefficients $c_{k}=a_{k}-i b_{k}$ of a function $p \in H^{1}$ are defined as in section 2.1. Let $F$ the set of $2 \pi$-periodic functions $p \in C^{1,1}(\mathbb{R}, \mathbb{R})$ which satisfy:

$$
\left\{\begin{array}{l}
p(\theta)=\frac{1}{2}+\sum_{k \geq 1} a_{2 k+1} \cos (2 k+1) \theta+b_{2 k+1} \sin (2 k+1) \theta, \\
p+p^{\prime \prime} \geq 0
\end{array}\right.
$$

and $F_{N}$ the subset of $F$ containing the trigonometric polynomials such that:

$$
\left\{\begin{array}{l}
p(\theta)=\frac{1}{2}+\sum_{1 \leq k \leq N} a_{2 k+1} \cos (2 k+1) \theta+b_{2 k+1} \sin (2 k+1) \theta \\
p+p^{\prime \prime} \geq 0
\end{array}\right.
$$

By compacity, there exists a minimum $p_{N}$ of $J$ in $F_{N}$ whose Fourier series is given for each $N$ by (33). Recall that $p_{R}$ denotes the support function of the Reuleaux triangle and $p_{R}$ is the unique minimizer of $J$ in $F$ (up to a translation) according to the Blaschke-Lebesgue theorem. Moreover, $\rho_{R}=p_{R}+p_{R}^{\prime \prime}$ is given by (14) and we have:

$$
p_{R}(\theta)=p_{R}(0) \cos \theta+p_{R}^{\prime}(0) \sin \theta+\int_{0}^{\theta} \sin (\theta-t) \rho_{R}(t) d t .
$$

As $F_{N} \subset F$, we have $A\left(p_{N}\right) \geq A\left(p_{R}\right)$ for all $N$. The aim of this section is to prove the following theorem.

Theorem 4.1. The sequence $p_{N}$ converges to $p_{R}$ in $H^{1}$ and $A\left(p_{N}\right)$ tends to $A\left(p_{R}\right)$ when $N$ tends to infinity.

The theorem is proved in two steps (Lemma 4.1 and Lemma 4.2). 
Remark 4.1. Let $\rho_{R}^{N}$ the partial sum of the Fourier series of $\rho_{R}$. By Fourier series theory, it is standard that $\rho_{R}^{N}$ converges to $\rho_{R}$ in the $L^{2}$-norm when $N$ goes to infinity and this convergence is not uniform as $\rho_{R}$ is discontinuous (Gibbs phenomenon). Moreover, the infimum of $\rho_{R}^{N}$ is strictly negative and converges to a certain value $\lambda<0$ (the exact value of $\lambda$ can be computed exactly in terms of the jump of $\rho_{R}$ at the discontinuity). As a consequence, no function $p_{R}^{N}$ solution of $p+p^{\prime \prime}=\rho_{R}^{N}$ belongs to $F_{N}$.

Lemma 4.1. Let $p_{N}$ a minimizer of $J$ in $F_{N}$. There exists $p \in F$ such that up to a subsequence, $p_{N}$ converges to $p$ in $H^{1}$. Moreover, $J\left(p_{N}\right)$ tends to $J(p)$ when $N$ tends to infinity.

Proof. Let $\alpha:=\inf _{N} A\left(p_{N}\right) \geq 0$. We have for all $N>0$ :

$$
\alpha \leq A\left(p_{N}\right)=\frac{\pi}{2}+\sum_{1 \leq k \leq N}\left(1-(2 k+1)^{2}\right)\left(a_{2 k+1}^{2}+b_{2 k+1}^{2}\right) .
$$

From this inequality, we deduce that $p_{N}$ is bounded in $H^{1}$, and consequently, there exist $p \in H^{1}$ such that up to a subsequence, $p_{N}$ weakly converges to $p$ in $H^{1}$. Moreover, by Cauchy-Schwarz inequality, there exists $C>0$ such that for all $N \geq 0$

$$
\sup _{\|\phi\|_{L^{2}} \leq 1}\left|\int_{0}^{2 \pi}\left(p_{N}+p_{N}^{\prime \prime}\right) \phi\right|=\sup _{\|\phi\|_{L^{2} \leq 1}}\left|\int_{0}^{2 \pi} p_{N} \phi-p_{N}^{\prime} \phi^{\prime}\right| \leq C .
$$

It follows that $\rho_{N}:=p_{N}+p_{N}^{\prime \prime}$ is bounded in $L^{2}$. Up to a subsequence, we may assume that $\rho_{N}$ weakly converges to a certain function $\rho \in L^{2}$. Let $(\alpha, \beta)$ be taken arbitrarily in $\mathbb{R}^{2}$ and set $p_{N}(0)=\alpha, p_{N}^{\prime}(0)=\beta$ for all $N>0$ (the area $A$ is invariant with respect to translations). We then have:

$$
p_{N}(\theta)=\alpha \cos \theta+\beta \sin \theta+\int_{0}^{\theta} \rho_{N}(t) \sin (\theta-t) d t,
$$

and

$$
p_{N}^{\prime}(\theta)=-\alpha \sin \theta+\beta \cos \theta+\int_{0}^{\theta} \rho_{N}(t) \cos (\theta-t) d t .
$$

By weak convergence of $\rho_{N}$, we obtain that for all $\theta \in[0,2 \pi]$ :

$$
p_{N}(\theta) \rightarrow \tilde{p}(\theta):=\alpha \cos \theta+\beta \sin \theta+\int_{0}^{\theta} \rho(t) \sin (\theta-t) d t
$$

Clearly, $\tilde{p}$ is of class $C^{1}$, and similarly, we get for all $\theta \in[0,2 \pi]$ :

$$
p_{N}^{\prime}(\theta) \rightarrow \tilde{p}^{\prime}(\theta):=-\alpha \sin \theta+\beta \cos \theta+\int_{0}^{\theta} \rho(t) \cos (\theta-t) d t .
$$

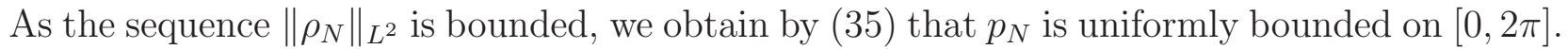
By Lebesgue's theorem, the sequence $p_{N}$ strongly converges to $p$ in $L^{2}$ and thus $\tilde{p}=p$. Using (36), we obtain similarly that $p_{N}^{\prime}$ strongly converges to $p^{\prime}$ in $L^{2}$. It remains to prove that $p$ is the support function of a planar constant width body. As for all $k \neq 0$ we have $c_{2 k}\left(p_{N}\right)=0$, we obtain at the limit $c_{2 k}(p)=0$. Moreover, for all non-negative function $\varphi$ of class $C^{2}$ with compact support on $[0,2 \pi]$, it holds

$$
\int_{0}^{2 \pi}\left(p+p^{\prime \prime}\right) \varphi=\lim _{N \rightarrow \infty} \int_{0}^{2 \pi}\left(p_{N}+p_{N}^{\prime \prime}\right) \varphi
$$


and the latter integral is non-negative as $p_{N} \in F_{N}$. Hence, $p+p^{\prime \prime} \geq 0$ and $p$ is in $F$. The convergence of $A\left(p_{N}\right)$ to $A(p)$ is a consequence of the convergence of $p_{N}$ to $p$ in $H^{1}$.

The next lemma is the main result and establishes the convergence in $H^{1}$ of a sequence $h_{N} \in F_{N}$ to $p_{R}$.

Lemma 4.2. There exists a sequence of trigonometric polynomials $h_{N} \in F_{N}$ converging to $p_{R}$ in $H^{1}$ and such that $A\left(h_{N}\right)$ tends to $A\left(p_{R}\right)$ when $N$ tends to infinity.

Proof. Let $\varepsilon>0$. We first prove the existence of a continuous periodic function $\rho_{\varepsilon}$, $C^{1}$-piecewise such that:

$$
\left\{\begin{array}{l}
\rho_{\varepsilon}(\theta)+\rho_{\varepsilon}(\theta+\pi)=1, \forall \theta \in \mathbb{R}, \\
\varepsilon \leq \rho_{\varepsilon}(\theta) \leq 1-\varepsilon, \\
\left\|\rho_{\varepsilon}-\rho_{R}\right\|_{L^{2}} \leq \sqrt{2} \varepsilon \\
\int_{0}^{2 \pi} \rho_{\varepsilon}(\theta) e^{i \theta} d \theta=0 .
\end{array}\right.
$$

There exists a continuous mapping $\bar{\rho}_{\varepsilon}:[0, \pi] \rightarrow \mathbb{R}, C^{1}$-piecewise such that:

$$
\left\{\begin{array}{l}
\varepsilon \leq \bar{\rho}_{\varepsilon}(\theta) \leq 1-\varepsilon, \forall \theta \in[0, \pi], \\
\int_{0}^{\pi}\left(\bar{\rho}_{\varepsilon}-\rho_{R}\right)^{2} \leq \varepsilon^{2} \\
\bar{\rho}_{\varepsilon}(0)=\bar{\rho}_{\varepsilon}(\pi)=\frac{1}{2} .
\end{array}\right.
$$

Notice that the restriction of $\rho_{R}$ to $[0, \pi]$ is symmetric with respect to $\theta=\frac{\pi}{2}$. It is therefore possible to assume that $\bar{\rho}_{\varepsilon}$ is also symmetric with respect to $\theta=\frac{\pi}{2}$ and that:

$$
\int_{0}^{\frac{\pi}{2}} \bar{\rho}_{\varepsilon}(\theta) \sin \theta d \theta=\frac{1}{2}
$$

By symmetry, $\bar{\rho}_{\varepsilon}$ satisfies:

$$
\int_{0}^{\pi} \bar{\rho}_{\varepsilon}(\theta) \cos \theta d \theta=0
$$

and by (41) one has:

$$
\int_{0}^{\pi} \bar{\rho}_{\varepsilon}(\theta) \sin \theta d \theta=1
$$

Let us now define $\rho_{\varepsilon}$ on $[0,2 \pi]$ by:

$$
\left\{\begin{array}{l}
\rho_{\varepsilon}(\theta)=\bar{\rho}_{\varepsilon}(\theta), \theta \in[0, \pi], \\
\rho_{\varepsilon}(\theta)=1-\bar{\rho}_{\varepsilon}(\theta-\pi), \theta \in[\pi, 2 \pi] .
\end{array}\right.
$$

By periodicity, the function $\rho_{\varepsilon}$ is extended to $\mathbb{R}$. By $(40)$, we have $\bar{\rho}_{\varepsilon}(0)=1-\bar{\rho}_{\varepsilon}(\pi)$, and consequently $\rho_{\varepsilon}$ is continuous for $\theta=\pi$ and $\theta=2 \pi$ and defines a continuous and $C^{1}$-piecewise mapping. By construction $\rho_{\varepsilon}$ takes values within $[\varepsilon, 1-\varepsilon]$ and satisfies $\rho_{\varepsilon}(\theta+\pi)+\rho_{\varepsilon}(\theta)=1$ for all $\theta \in \mathbb{R}$. Now we have by (42) and (43):

$$
\int_{0}^{2 \pi} \rho_{\varepsilon}(\theta) e^{i \theta} d \theta=2 \int_{0}^{\pi} \bar{\rho}_{\varepsilon}(\theta) e^{i \theta} d \theta-2 i=0
$$

Finally:

$$
\left\|\rho_{\varepsilon}-\rho_{R}\right\|_{L^{2}}^{2}=2 \int_{0}^{\pi}\left(\bar{\rho}-\rho_{R}\right)^{2} \leq 2 \varepsilon^{2}
$$


and we get (39).

Now consider $q_{N}$ the partial sum of the Fourier series of $\rho_{\varepsilon}$. As $\rho_{\varepsilon}$ is continuous and $C^{1}$ piecewise, $q_{N}$ uniformly converges to $\rho_{\varepsilon}$ on $\mathbb{R}$, and there exists $N_{0}$ such that for all $N \geq N_{0}$ :

$$
\left\|q_{N}-\rho_{\varepsilon}\right\|_{L^{\infty}} \leq \frac{\varepsilon}{2}
$$

Thus, $q_{N}$ takes values within $\left[\frac{\varepsilon}{2}, 1-\frac{\varepsilon}{2}\right]$ for $N \geq N_{0}$, and by the triangular inequality, one has:

$$
\left\|q_{N}-\rho_{R}\right\|_{L^{2}} \leq \beta \frac{\varepsilon}{2}
$$

where $\beta=\sqrt{2}+\pi$. To finish the proof, let us define $h_{N}$ as the solution of $h_{N}+h_{N}^{\prime \prime}=q_{N}$ such that:

$$
h_{N}(\theta)=p_{R}(0) \cos \theta+p_{R}^{\prime}(0) \sin \theta+\int_{0}^{\theta} \sin (\theta-t) q_{N}(t) d t .
$$

A standard computation shows that:

$$
h_{N}^{\prime}(2 \pi)-h_{N}^{\prime}(0)-i\left(h_{N}(2 \pi)-h_{N}(0)\right)=\int_{0}^{2 \pi} q_{N}(\theta) e^{i \theta} d \theta .
$$

By (39) the first Fourier coefficient of $\rho_{\varepsilon}$ is equal to zero, therefore the last quantity is equal to zero which ensures that $h_{N}$ is $2 \pi$-periodic. By construction $0 \leq q_{N}=h_{N}+h_{N}^{\prime \prime} \leq 1$ and we have for all $\theta \in \mathbb{R}: h_{N}(\theta)+h_{N}(\theta+\pi)=1$ (by the decomposition of $q_{N}$ on the harmonics of order $2 k+1$ ). Thus, $h_{N}$ belongs to $F_{N}$. By using (34) and the Cauchy-Schwarz inequality, we get:

$$
\left\|h_{N}-p_{R}\right\|_{L^{2}} \leq \pi \beta \varepsilon
$$

and consequently $h_{N}$ converges to $p_{R}$ in $L^{2}$. Similarly, $h_{N}^{\prime}$ converges to $p_{R}^{\prime}$ in $L^{2}$. Finally, $A\left(h_{N}\right)$ tends to $A\left(p_{R}\right)$ when $N$ tends to infinity which ends the proof of the Lemma.

Proof of theorem 4.1. By Lemma 4.1, the sequence of minimizers $p_{N}$ converges to $p \in F$ in $H^{1}$. As $h_{N} \in F_{N}$, we have:

$$
A\left(p_{R}\right) \leq A\left(p_{N}\right) \leq A\left(h_{N}\right) .
$$

From Lemma 4.2, $A\left(h_{N}\right)$ tends to $A\left(p_{R}\right)$. Hence, $A\left(p_{N}\right)$ tends to $A(p)=A\left(p_{R}\right)$ and by unicity of the minimizer of $A$ in $F$, we obtain $p(\theta)=p_{R}(\theta)+\left(\alpha-p_{R}(0)\right) \cos \theta+\left(\beta-p_{R}^{\prime}(0)\right) \sin \theta$, $\forall \theta \in \mathbb{R}$.

Remark 4.2. The result of Theorem 4.1 can be extended in the case of planar rotors of a regular polygon $P_{n}$. The width constraint is replaced by (19), and the Fourier decomposition of a rotor involves harmonics of orders $k n+1$ and $k n-1$. The proof mimics that of Lemma 4.2. We believe that the result of Theorem 4.1 can be transposed to the problem of section 3.3. Although the admissible set in section 3.3 is a subset of $F_{N}$, the functional is non-autonomous and the proof must be modified.

\section{Conclusion and perspectives}

This paper aims at giving a numerical approach to investigate optimization problems in the class of convex bodies. As far as we know, the transformation of the convexity constraint into a semidefinite programming problem has not been previously studied in this framework. 
This method seems to be efficient in the context of planar constant width bodies and rotors, even though minimization of a concave functional on a convex set is a difficult problem in principle. The approximation can be made precise quickly (a few seconds on a standard PC) by increasing the number of Fourier coefficients to more than 30 .

We believe this work opens a promising way to investigate other optimization problems in the class of planar and space convex bodies. To extend this method in $\mathbb{R}^{3}$ the Fourier series decomposition must be replaced by bivariate spherical harmonics. Nevertheless, the decomposition of non-negative spherical harmonics into sum-of-squares is more subtle in this case. The question of finding a numerical relevant method to investigate Meissner's conjecture [6] on the constant breadth body of minimal volume remains a challenging problem, as recalled in [12].

\section{Acknowledgments}

We would like to thank Jean-Baptiste Hiriart-Urruty for many fruitful discussions and for being at the origin of our collaboration. The second author acknowledgdes support by project No. 103/10/0628 of the Grant Agency of the Czech Republic.

\section{References}

[1] J. A. Andrejewa, R. Klötzler, Zur analytischen Lösung geometrischer Optimierungsaufgaben mittels Dualität bei Steuerungsproblemen. Teil I, Zeitschrift für angewandte Mathematik und Mechanik, vol. 64, no. 1, pp. 35-44, 1984.

[2] J. A. Andrejewa, R. Klötzler, Zur analytischen Lösung geometrischer Optimierungsaufgaben mittels Dualität bei Steuerungsproblemen. Teil II, Zeitschrift für angewandte Mathematik und Mechanik, vol. 64, no. 1, pp. 147-153, 1984.

[3] T. Bayen, Optimisation de formes dans la classe des corps de largeur constante et des rotors, Thèse de Doctorat, Université Paris 6, 2007.

[4] T. Bayen, Analytic Parametrization of Rotors and Proof of a Goldberg Conjecture by Optimal Control Theory, SIAM J. Control Optim., vol. 47, pp. 3007-3036, 2008.

[5] T. Bayen, Parametrization of a Convex Optimization Problem by Optimal Control Theory and Proof of a Goldberg Conjecture, Proc. 48th IEEE Conf. on Decision and Control, 2009 .

[6] T. Bayen, É. Oudet, T. Lachand-Robert, Analytic parametrization and minimization of the volume of orbiforms, Arch. Ration. Mech. Anal., vol. 146, pp. 225-249, 2007.

[7] B. Dumitrescu, Positive Trigonometric Polynomials and Signal Processing Applications, Springer Verlag, Berlin, 2007.

[8] W. J. Firey, Isoperimetric ratios of Reuleaux polygons, Pacific J. Math., vol. 10, pp. 823-829, 1960. 
[9] M. Goldberg, Trammel rotors in regular polygons, Amer. Math. Monthly, vol. 64, pp. 71-78, 1957.

[10] E. Harrell, A direct proof of a theorem of Blaschke and Lebesgue, J. Geom. Anal., vol. 12 , pp. 81-88, 2002.

[11] Evans M. Harrell II, A. Henrot, On the maximization of functionals on convex regions, and the characterization of the farthest convex set, Mathematiki, to appear, 2010.

[12] J.-B. Hiriart-Urruty, An open global optimization problem. J. Global Opt., vol. 45, no. 2, pp. 335-336, 2009.

[13] F. Malagoli, An optimal control theory approach to the Blaschke-Lebesgue theorem, J. Convex Anal., vol. 16, no. 2, pp. 391-407, 2009.

[14] Yu. Nesterov, Squared Functional Systems and Optimization Problems, Chapter 17, pp. 405-440 in H. Frenk, K. Roos, T. Terlaky and S. Zhang (Editors), High Performance Optimization, Kluwer Academic Publishers, Dordrecht, The Netherlands, 2000.

[15] D. Henrion, J. Löfberg, M. Kočvara, M. Stingl. Solving polynomial static output feedback problems with PENBMI. Proc. of joint IEEE Conf. on Decision and Control and Europ. Control Conf., Seville, Spain, 2005.

[16] T. Lachand-Robert, M. A. Peletier, Newton's Problem of the Body of Minimal Resistance in the Class of Convex Developable Functions, Math. Nachr. vol. 226, pp. 153-176, 2001.

[17] J. Lamboley, M. Pierre, Structure of shape derivatives around irregular domains and applications, Journal of Convex Analysis, vol. 14, no. 4, pp. 807-822, 2007.

[18] J. Löfberg. YALMIP: a toolbox for modeling and optimization in Matlab. Proc. IEEE Symp. on Computer-Aided Control System Design, Taipei, Taiwan, 2004.

[19] S. Rabinowitz, A polynomial curve of constant width, Missouri J. Math. Sci., vol. 9, pp. 23-27, 1997.

[20] R. Schneider, Convex Bodies - the Brunn-Minkowski Theory, Cambridge University Press, 1993.

[21] I. M. Yaglom, V. G. Boltyanski, Convex Figures, Gosudarstv. Izdat. Tehn.-Teor. Lit., Moscow-Leningrad, 1951. 